Meunier et al.

\title{
Gravity-based microfiltration reveals unexpected prevalence of circulating tumor cell clusters in ovarian cancer
}

\author{
Anne Meunier, ${ }^{1,2}$ Javier Alejandro Hernández-Castro, ${ }^{1,2,3}$ Sara Kheireddine, ${ }^{1,2}$ Sara Al Habyan,,5 \\ Benjamin Péant, ${ }^{6}$ Teodor Veres, ${ }^{1,3}$ Luke McCaffrey, ${ }^{4,5,7}$ Diane Provencher, ${ }^{6,8}$ Anne-Marie Mes- \\ Masson, ${ }^{6,9}$ and David Juncker ${ }^{1,2,10^{*}}$
}

\footnotetext{
Affiliations

${ }^{1}$ Biomedical Engineering Department, McGill University, Montreal, QC, H3A 2B4, Canada

${ }^{2}$ McGill University \& Genome Quebec Innovation Centre, McGill University, Montreal, QC, H3A 0G1, Canada

${ }^{3}$ National Research Council of Canada, QC, J4B 6Y4, Canada

${ }^{4}$ Rosalind and Morris Goodman Cancer Research Centre, McGill University, Montreal, QC, H3A 1A3, Canada

${ }^{5}$ Division of Experimental Medicine, McGill University, Montreal, QC, H4A 3J1, Canada

${ }^{6}$ Institut du cancer de Montréal and Centre de recherche du Centre hospitalier de l'Université de Montréal

(CRCHUM), QC, H2X 0A9, Canada

${ }^{7}$ Gerald Bronfman Department of Oncology, McGill University, Montreal, QC, H4A 3T2, Canada

${ }^{8}$ Division of Gynecologic Oncology, Department of Obstetrics-Gynecology, Université de Montréal, Montreal, QC H3T 1J4, Canada

${ }^{9}$ Department of Medicine, Faculty of Medicine, Université de Montréal, Montréal, QC H3T 1J4, Canada

${ }^{10}$ Neurology and Neurosurgery Department, McGill University, Montreal, H3A 2B4, QC, Canada

*Correspondence: david.juncker@mcgill.ca
} 
Meunier et al.

\section{Summary}

Circulating tumor cells (CTCs) are rare (few cells per milliliter of blood) and mostly isolated as single cell CTCs (scCTCs). CTC clusters (cCTCs), even rarer, are of growing interest, notably because of their higher metastatic potential, but very difficult to isolate. Here, we introduce gravity-based microfiltration $(\mathrm{G} \mu \mathrm{F})$ for facile isolation of cCTCs. We identify cluster break-up as a confounding cause, and achieve $\sim 85 \%$ capture efficiency. $\mathrm{G} \mu \mathrm{F}$ from orthotopic ovarian cancer mouse models and from 10 epithelial ovarian cancer (EOC) patients uncovered cCTCs in every case, with between 2-100+ cells. cCTCs represented between $5-30 \%$ of all CTC events, and 10-80\% of captured CTCs were clustered; remarkably, in two patients, more CTCs were circulating as cCTCs than scCTCs. G $\mu$ F uncovered the unexpected prevalence, frequency and sometimes large size of cCTCs in EOC patients with either metastatic and localized disease, and motivates additional studies to uncover their properties and role in disease progression.

\section{Keywords}

Circulating tumor cells (CTCs), clusters of circulating tumor cells (cCTCs), CTC enrichment, gravitybased microfiltration $(\mathrm{G} \mu \mathrm{F})$, epithelial ovarian cancer (EOC), epithelial-mesenchymal transition (EMT) 
Meunier et al.

\section{Introduction}

Circulating tumor cells (CTCs) are shed from the tumor into the bloodstream, then circulate and reach distant locations where they can implant, proliferate and give rise to metastases. CTCs, found in the majority of epithelial cancers (Pantel and Speicher, 2016), represent a crucial intermediate in the metastatic cascade and their study has the potential to help improve patient care. CTCs are extremely rare ( 1-10 CTCs vs. $<10^{6}$ leukocytes and $<10^{9}$ erythrocytes per milliliter of blood) and display extensive molecular heterogeneity. The first report on CTCs dates back to 1869 (Ashworth, 1869), however interest in CTCs as predictive and prognostic markers has dramatically increased over the past decades, following technical advances permitting their isolation and quantification. It has been demonstrated that CTCs in blood are correlated with poor outcome, recurrence and resistance to therapy (Pantel and Speicher, 2016, Poveda et al., 2011).

CTCs exist as single cells (scCTCs) and multicellular aggregates, called CTC clusters (cCTCs). scCTCs were traditionally isolated and identified based on the expression of the Epithelial Cell Adhesion Molecule (EpCAM) and cytokeratins (CK), but it is now known that many scCTCs are disseminated following epithelial-mesenchymal transition (EMT) leading to the downregulation of epithelial markers and resulting in their enhanced motility and aggressiveness (Pantel and Speicher, 2016). cCTCs do not arise from scCTC proliferation in blood but separate from the primary tumor as a cluster (collective dissemination) (Aceto et al., 2014). Using mouse models, Aceto et al. found that cCTCs accounted for $3 \%$ of all captured CTC events, but have a 23- to 50-time higher metastatic potential than scCTCs (Aceto et al., 2014). The presence of cCTCs in blood has been associated with worse outcome in patients with lung, breast, prostate, skin, and ovarian cancer (Au et al., 2016, Sarioglu et al., 2015, Pantel and Speicher, 2016, Aceto et al., 2014).

In epithelial ovarian cancer (EOC), CTCs were detected in the blood of $\sim 10$ to $\sim 60 \%$ patients (Judson et al., 2003, Fan et al., 2009), travelling as scCTCs (Allard et al., 2004) and rarely as cCTCs (Guo et al., 2018). EOC is also characterized by the formation of ascites within the peritoneal cavity, that contain tumor cells and provide a local microenvironment regulating the behavior of scCTCs and cCTCs (Kim et al., 2016). Particularly observed in advanced high-grade serous carcinomas (HGSC), peritoneal dissemination constitutes the primary route of metastasis in EOC, and is associated with disease recurrence (Kim et al., 2016). In line with observations of Aceto et al. in blood, some of the present authors showed that CTCs in ascites of EOC mouse models arise from spontaneous detachment as either single cells or clusters and, although scCTCs still constituted the majority of all CTC events in ascites, 
Meunier et al.

cCTCs represented 17-49\% of all CTC events in ascites (Al Habyan et al., 2018). Enriching cCTCs is therefore of main interest as their characterization could offer new insights into cancer dissemination, and help improve prognosis and treatments in cancer.

Isolating cCTCs is challenging given their rarity, their short lifespan, and their propensity to disaggregate under shear (Sarioglu et al., 2015). Isolation technologies were initially developed and optimized for scCTCs, and following the occasional capture of cCTCs (Brandt et al., 1996, Reddy et al., 2016, Stott et al., 2010), efforts were directed towards isolating clusters with better efficiency and selectivity (Ferreira et al., 2016). Toner and colleagues pioneered two methods for selective cCTC isolation, including a deterministic lateral displacement system that deflects particles $>30 \mu \mathrm{m}$ and effectively isolated artificial breast cancer cell clusters spiked in blood, but was not yet tested on patient samples (Au et al., 2017). They also developed a filtration-based chip (Cluster-chip) using shifted triangular pillars forming 4000 parallel $12 \times 100 \mu \mathrm{m}^{2}$ slit openings designed to capture cCTCs, while allowing scCTCs to pass through. Artificial clusters spiked into blood were captured with a yield of $100 \%$ for sizes of 4-cells and above at a flow rate of $0.04 \mathrm{~mL} \mathrm{~min}^{-1}$. The Cluster-chip was used with blood from metastatic breast, prostate, and skin cancer, and cCTCs with 2-19 cells were isolated from as many as 30-40\% patients, thus constituting to the best of our knowledge the highest capture rate for cCTC reported to date (Sarioglu et al., 2015).

However, common limitations of CTC technologies are amplified for cCTCs. Indeed, microfluidic methods suffer from limited throughput and capacity, and involve long times for processing the standard 5-7 mL of patient blood, which diminishes the viability of isolated cells (Tan et al., 2009). cCTCs, being at risk of dissociation, are more susceptible to shear stress, which must be reduced at the cost of even lower flow rates. New methods are being developed and for instance, Cheng et al. used a threedimensional scaffold with low shear stress, capturing both scCTCs and cCTCs at $50 \mu \mathrm{L} \mathrm{min}^{-1}$, followed by their release, which appears efficient, but may lead to aggregation and disaggregation that could confound the results (Cheng et al., 2017).

Microfiltration is geometrically predisposed to generate low shear stresses because circular filters accommodate tens of thousands of pores, each forming a parallel flow path with a very low shear stress. Microfiltration of scCTC was first reported in 1964 (Seal, 1964), using track-etched membranes which, owing to a random pore distribution that often limits porosity to $<5 \%$, impose low throughput (Fleischer et al., 1964, Holdich et al., 2006). Interestingly, the use of such microfilters for cCTC isolation has recently been reported by Sarioglu et al. using $5 \mu \mathrm{m}$-diameter pores, but with very low yield (Sarioglu et 
Meunier et al.

al., 2015). Following advances in microfabrication, membranes with regular pore distribution thus high porosity were made, enabling higher flow rates while maintaining low shear stress (Adams et al., 2014, Ferreira et al., 2016, Lim et al., 2012). We recently developed high performance polymer microfilters that meet the major criteria for practical use, namely low-cost, durable, highly transparent, nonautofluorescent and highly porous (20-60\%) (Hernandez-Castro et al., 2017). Following optimization, 8 mm-diameter microfilters with $8 \mu \mathrm{m}$ pores, a 1:6 dilution of whole blood in phosphate buffer saline (PBS), and a flow rate of $0.1 \mathrm{~mL} \mathrm{~min}^{-1}$ set using programmable syringe pumps allowed us to recover $>$ $80-95 \%$ of cancer cells spiked into blood (Meunier et al., 2016).

Here, we introduce gravity-based microfiltration $(\mathrm{G} \mu \mathrm{F})$ for the efficient isolation of cCTCs using the inhouse fabricated microfilters described above (Hernandez-Castro et al., 2017), and 3D printed cartridges (Meunier et al., 2016). First, the gravity-flow of buffer, diluted and whole blood using filters with pores ranging from 8 to $28 \mu \mathrm{m}$ in diameter was characterized, and flow rates, consistent with predictions, measured. The optimal flow rate and pore size for cCTCs isolation were determined using blood from healthy donors spiked with OV-90 ovarian cancer cells comprising both single cells and clusters. Following a systematic characterization of the cluster morphology, the effect of shear on cluster disaggregation was discovered and quantified. Next, blood and ascites from ovarian orthotopic transplanted mice were processed and the number of scCTCs and cCTCs, along with the morphological features and the expression of molecular markers for aggressiveness and EMT were evaluated. Finally, blood samples from 10 EOC patients with advanced (metastatic) and localized disease, chemo-naïve and post-chemotherapy were analyzed, uncovering an unexpectedly high prevalence and heterogeneity of cCTCs in all cancer sub-types.

\section{Results}

\section{Gravity-based microfiltration for selective isolation of clustered CTCs}

Filter clogging by blood components is expected to increase the pressure and shear stress across the pores under constant flow rate conditions. We reasoned that increased shear would negatively affect the capture of clusters as it could contribute to disrupting or squeezing them through the pores. Thus we adopted gravity-based microfiltration $(\mathrm{G} \mu \mathrm{F})$, which provides constant pressure with constant total column heights $\left(\mathrm{H}_{\mathrm{tot}}\right)$ of 15, 20, and $30 \mathrm{~cm}$ (Figures 1A and S1) (Ripperger et al., 2012). As before, blood was diluted 
Meunier et al.

1:6 in PBS and an $8 \mathrm{~mm}$-diameter microfilter was clamped in the 3D printed cartridge (Figure 1B). Filters with pores of $8 \mu \mathrm{m}$ diameter (Table S1) were used initially to characterize flow of G $\mu \mathrm{F}$ using PBS, diluted blood, and whole blood.

For all samples, flow rate quickly decreased during the first seconds to minutes (Domain I), then, for PBS, flow rate decreased in concordance with the column height (Domain II) until the end of filtration (Figures 1C, S1B and Table S2). For diluted and whole blood, initial flow rates also quickly decreased in domain I, then stabilized in domain II, which could last hours (Figures 1D and S1C-D). For example, for $\mathrm{H}_{\text {tot }}=20 \mathrm{~cm}\left(\mathrm{~L}_{1}=10 \mathrm{~cm}\right)$, the flow rate in domain II was $\sim 0.09$ and $\sim 0.02 \mathrm{~mL} \mathrm{~min}^{-1}$ for diluted blood and whole blood, respectively. For both fluids, the flow rate in domain II varied linearly with $\mathrm{H}_{\text {tot. }}$ For diluted blood, similar behavior was observed for all filter pore sizes, and calibration curves (Figure S2), allowed the determination of the tube length to use for achieving a specific flow rate (Table S1). 
Meunier et al.
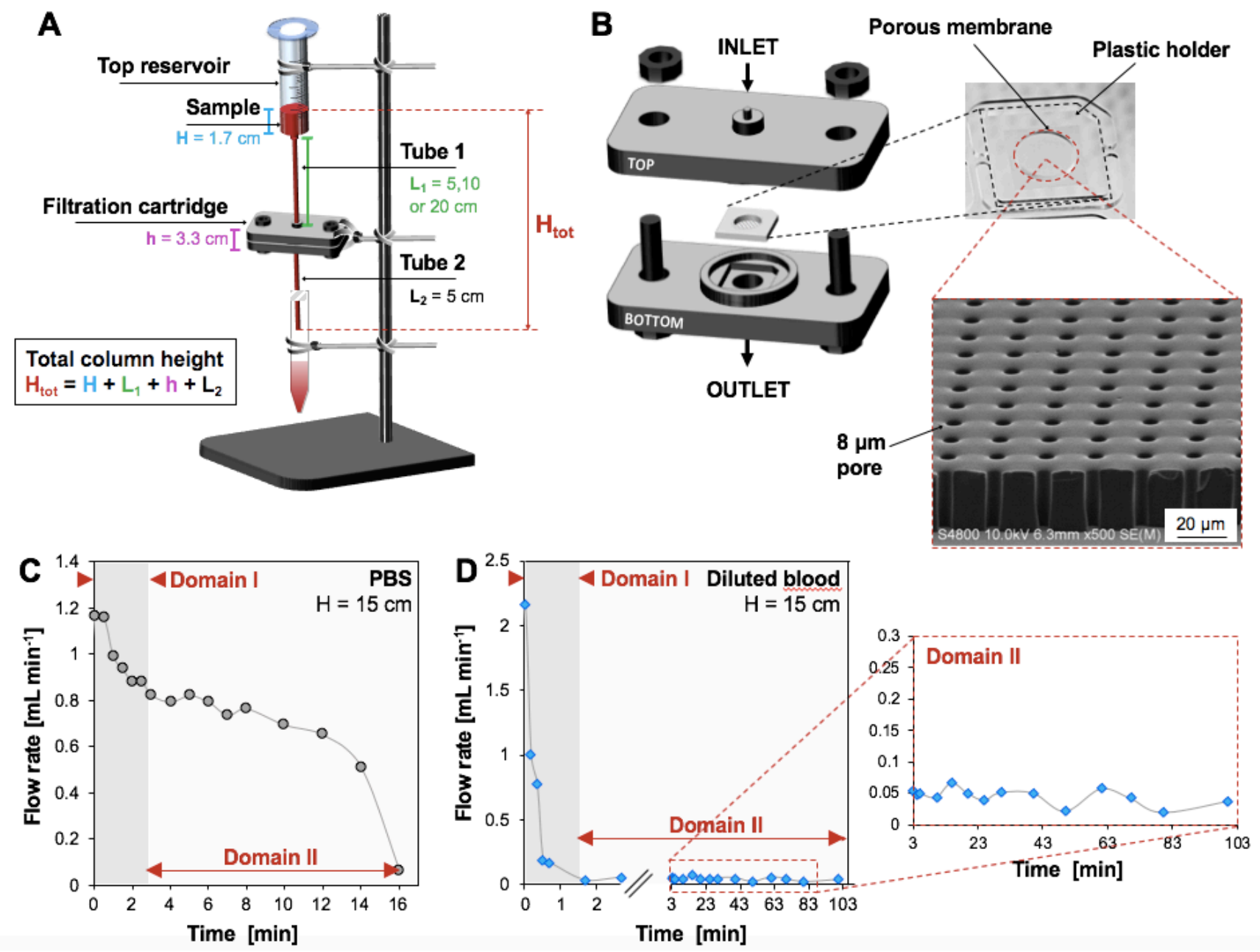

Figure 1. G $\boldsymbol{\mu F}$ for CTC enrichment. (A) Schematic of the $\mathrm{G} \mu \mathrm{F}$ set-up. The total column height $\left(\mathrm{H}_{\text {tot }}\right)$ determines the pressure and flow rate when considering the $\mathrm{G} \mu \mathrm{F}$ flow resistance. (B) Exploded view of the filtration cartridge with filter, and close-ups of a polymer filter with $8 \mu \mathrm{m}$ diameter pores. (C) and (D) Examples of time course of flow rate for $10 \mathrm{~mL}$ of (C) PBS and (D) diluted blood filtered through an $8 \mu \mathrm{m}$ filter with $\mathrm{H}_{\text {tot }}=15 \mathrm{~cm}$, and closeup of domain II (pseudo-steady state) for diluted blood. See also Figures S1 and S2 and Tables S1 and S2.

To assess whether small differences between $\mathrm{G} \mu \mathrm{F}$ and syringe pump driven flow were significant, diluted blood samples were spiked with OV-90 ovarian cancer single cells and clusters, divided into two equal volumes and filtered through $8 \mu \mathrm{m}$ filters using both methods: one by $\mathrm{G} \mu \mathrm{F}$ with a tube length $\mathrm{L}_{1}$ of 12 $\mathrm{cm}$ and $66 \mathrm{~cm}$ (nominal flow rates of $\sim 0.1$ and $\sim 0.5 \mathrm{~mL} \mathrm{m^{-1 }}$, Table $\mathrm{S} 1$ ), and the other using a syringe pump with exactly 0.1 and $0.5 \mathrm{~mL} \mathrm{~min}^{-1}$. For $8 \mu \mathrm{m}$ filters, the calculated transmembrane pressure $(\Delta \mathrm{P})$ was 5.2 $\mathrm{Pa}$ and 26.1 Pa, respectively (Table S3). After filtration, filters were rinsed with PBS, then cells 
Meunier et al.

were stained with DAPI for the nucleus, for epithelial cytokeratin (CK), and for CD45 to discriminate CTCs $\left(\mathrm{CK}^{+} / \mathrm{CD}^{-} / \mathrm{DAPI}^{+}\right)$from $\mathrm{WBCs}\left(\mathrm{CK}^{-} / \mathrm{CD}^{\circ} 5^{+} / \mathrm{DAPI}^{+}\right)$(Figure $\left.2 \mathrm{~A}\right)$.

The capture efficiency for single cells and clusters showed opposing trends (Figure 2B). When the flow rate increased from 0.1 to $0.5 \mathrm{~mL} \mathrm{~min}^{-1}$, cluster capture efficiency decreased from $\sim 80 \%$ to $\sim 40 \%$ for $\mathrm{G} \mu \mathrm{F}$, and from $\sim 35 \%$ to $\sim 25 \%$ for pump-controlled flow, while single cell capture increased from $60 \%$ to $200 \%$ for $\mathrm{G} \mu \mathrm{F}$, and from $55 \%$ to $230 \%$ for pump filtration. Percentages over $100 \%$ are attributed to the break-up of clusters, which can account for the contradicting trends. These results highlight that $\mathrm{G} \mu \mathrm{F}$ is more efficient at capturing clusters, in both cases, and that increasing the flow rate broke up 50\% of clusters for $\mathrm{G} \mu \mathrm{F}$, and only $\sim 33 \%$ of clusters for syringe flow. This discrepancy may be explained by the fact that syringe flow already disrupted clusters at low flow rate.

Next, the size of OV-90 clusters after filtration at a nominal rate of $0.1 \mathrm{~mL} \mathrm{~min}^{-1}$ for $\mathrm{G} \mu \mathrm{F}$ and syringe pump filtration was compared (Figure 2C). Consistent with cluster breakage, pump filtration showed more 2-cell clusters, but less 3-cell clusters, while 4-cell clusters and larger were essentially absent $(\sim 1 \%$ of all capture events). Clusters of all sizes were captured by $\mathrm{G} \mu \mathrm{F}$, and 4-cell clusters and larger collectively accounted for $\sim 30 \%$ of all captured events.

Previous optimization showed that the number of clogged pores after filtration and rinsing was negligible $(\sim 1 \%)$ (Meunier et al., 2016). However, it is most likely that a significant number of WBCs is depleted during the rinse step. The proportion of clogged pore might be higher and vary during filtration. In $\mathrm{G} \mu \mathrm{F}$, although flow rate remained stable overall in domain II, the slight fluctuations might reflect pore clogging, which would result in a slight pressure increase with constant flow rate pump filtration and explain the higher breaking rate. At $0.1 \mathrm{~mL} \mathrm{~min}^{-1}$, the maximum flow speed $\left(v_{\max }\right)$ through $8 \mu \mathrm{m}$ pores was $827 \mu \mathrm{m} \mathrm{s}^{-1}$. For $\mathrm{G} \mu \mathrm{F}, v_{\max }$ remained constant irrespective of pore clogging but increased under constant-flow rate conditions during syringe pump filtration as shown by the FEA in figure 2D. With 8 $\mu \mathrm{m}$ filter, for $22 \%$ clogging, $v_{\max }$ increased to $\sim 1060 \mu \mathrm{m} \mathrm{s}^{-1}$ and the resulting shear stress $\left(\tau_{\max }\right)$, from 1.59 to 2.05 (Table S3). 
Meunier et al.

A Single cells and small clusters
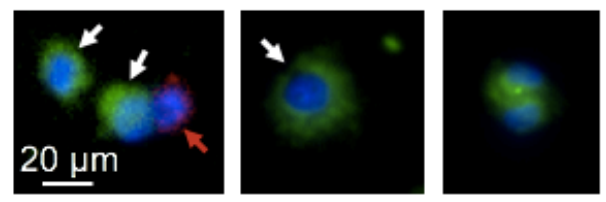

Large clusters
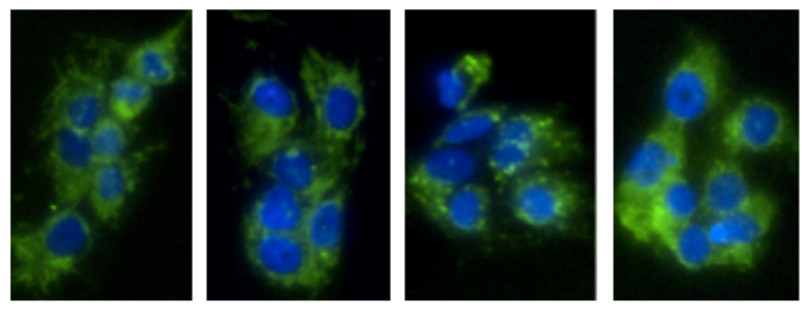

CK, CD45, DAPI
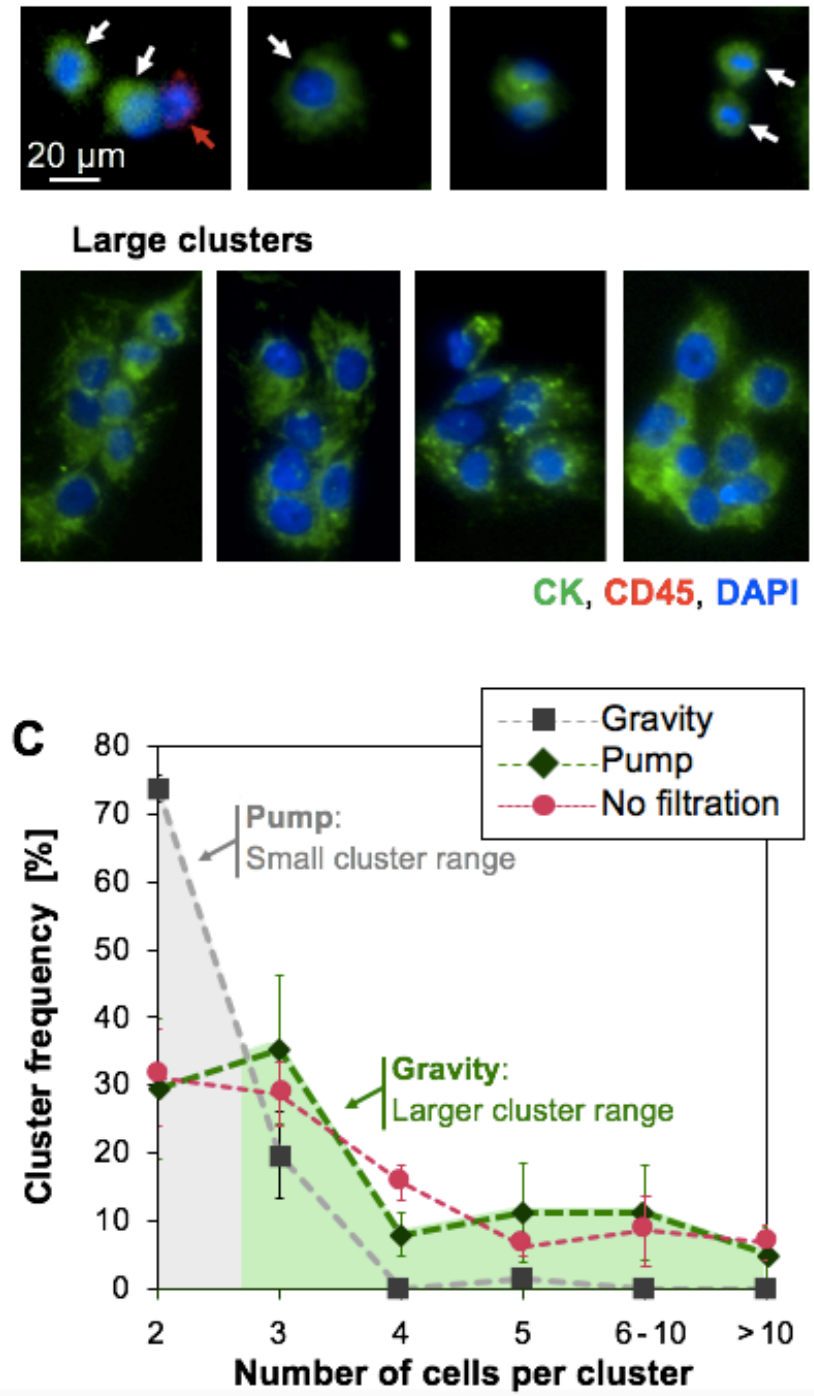

B

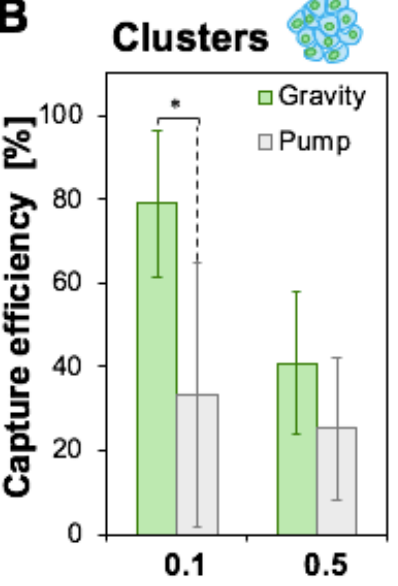

Flow rate $\left[\mathrm{mL} \mathrm{min}^{-1}\right]$

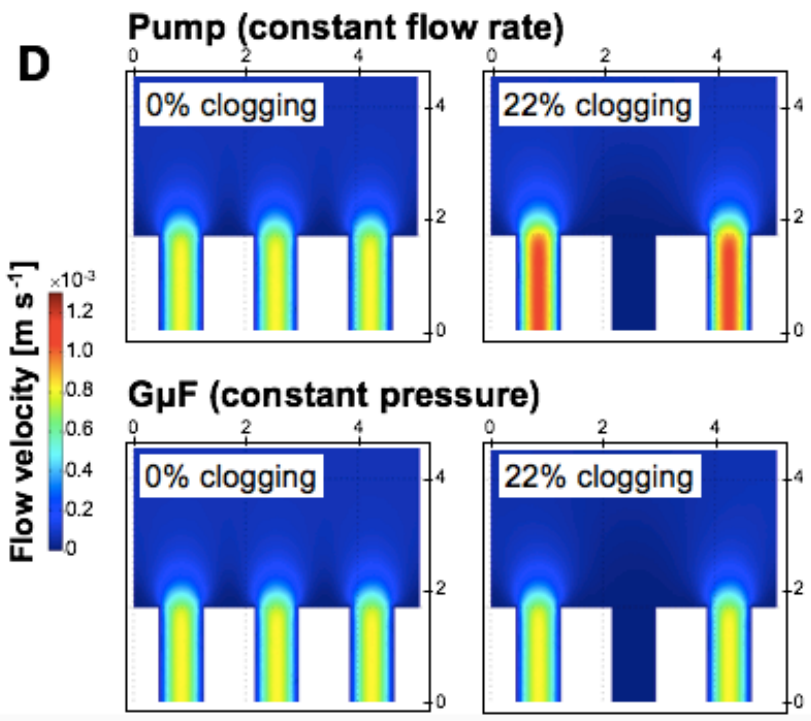

Figure 2. G $\mu \mathrm{F}$ captured larger clusters than syringe-pump driven flow. (A) Fluorescence images of OV-90 single cells and clusters captured using $\mathrm{G} \mu \mathrm{F}$ and pump filtration using $8 \mu \mathrm{m}$ filters. Cells were stained for cytokeratin (green) and CD45 (red). Nuclei were stained with DAPI (blue). Single cells (white arrows), small clusters and WBCs (red arrow) were captured using both configurations. Larger clusters, with > 5-6 cells were only captured with $\mathrm{G} \mu \mathrm{F}$. (B) Capture efficiency of clusters and single cells for $\mathrm{G} \mu \mathrm{F}$ (green) and pump filtration (grey) at 0.1 and $0.5 \mathrm{~mL} \mathrm{~min}^{-1}$. (C) Size distribution of the clusters before filtration and captured using G $\mu \mathrm{F}$ and pump filtration at $\sim 0.1 \mathrm{~mL} \mathrm{~min}^{-1}$. For each replicate, a known number of OV-90 cells ( $\sim 120$ clusters and $\sim 40$ single cells) was spiked in diluted blood. Error bars correspond to the standard deviation of three replicated experiments.

(D) Finite-element analysis comparing flow velocity in $8 \mu \mathrm{m}$ pores for $\mathrm{G} \mu \mathrm{F}$ and pump filtration at $0.1 \mathrm{~mL} \mathrm{~min}^{-}$ ${ }^{1}$, for $0 \%$ and $22 \%$ clogging. See also Tables S1 and S3. 
Meunier et al.

\section{Capture of OV-90 ovarian cancer cell line clusters}

Next, again using OV-90 cells, the optimal filter pore size for capturing the largest range of clusters was determined. Following spiking with $\sim 150$ single cells and $\sim 100$ clusters, each sample was divided in six and flowed through filters with $8 \mu \mathrm{m}, 10 \mu \mathrm{m}, 12 \mu \mathrm{m}, 15 \mu \mathrm{m}, 20 \mu \mathrm{m}$ and $28 \mu \mathrm{m}$ pores, respectively, using tube length $\mathrm{L}_{1}$ of $12,11,7,6,6$ and $6 \mathrm{~cm}\left(\sim 0.1 \mathrm{~mL} \mathrm{~min}^{-1}\right)$ (Figure 3A and 3B). Single OV-90 cells were found on all filters and single cell capture efficiency strongly decreased with increasing pore size, while matching the size distribution of OV-90 cell before filtration, ranging between $\sim 6-50 \mu \mathrm{m}$ in diameter. $\sim 70 \%$ of single cells were captured on $10 \mu \mathrm{m}$ filters, dropping to $\sim 25 \%$ and $2 \%$ for 15 and $28 \mu \mathrm{m}$ filters, respectively. Clusters were also found on all filters, and efficiency also decreased with pore size, but at a different rate and the efficiency was still $\sim 7 \%$ for $15 \mu \mathrm{m}$ pores. Increasing pore size also yielded higher purity, with decreasing number of contaminating WBCs from 1000 to $200 \mathrm{WBC}$ following filtration of $1 \mathrm{~mL}$ using $8 \mu \mathrm{m}$ and $15 \mu \mathrm{m}$ filters, respectively (Meunier et al., 2016).

Next, diluted blood samples spiked with OV-90 clusters were filtered successively through all filters by order of decreasing porosity and the cluster size distribution on each filter was determined by counting the number of cells they comprised and by measuring their surface area (Figure 3C and S3A). In this repeated filtration, all large clusters, from 6-100+ cells $\left(>1200 \mu \mathrm{m}^{2}, \varnothing_{\mathrm{eq}} \sim 40 \mu \mathrm{m}\right)$, were captured on the three first filters with 28,20 and $15 \mu \mathrm{m}$ pores. As expected, $28 \mu \mathrm{m}$ filters allowed for capturing the largest clusters with area $\sim 65000 \mu \mathrm{m}^{2}\left(\varnothing_{\mathrm{eq}} \sim 90 \mu \mathrm{m}\right)$ but most of the cluster population was captured on the following $20 \mu \mathrm{m}$ and $15 \mu \mathrm{m}$ filters, where cluster with area up to $\sim 2800 \mu \mathrm{m}^{2}$ and $\sim 5500 \mu \mathrm{m}^{2}$ were found, respectively. Small pore filters $(8,10$, and $12 \mu \mathrm{m})$ mostly captured 2 - and 3-cell clusters with areas $\sim 150$ $300 \mu \mathrm{m}^{2}\left(\emptyset_{\mathrm{eq}} \sim 14-19 \mu \mathrm{m}\right)$, that could pass through the preceding larger pore filters. Small clusters were also found on larger pore size filters but to a lesser extent. Interestingly we observed that in small clusters (up to 5-6 cells), the diameter of the biggest cell increased with the pore size (Figure S3B). These results suggest that the capture efficiency of small clusters may not be dictated solely by the size of the cluster, but significantly depends on the size of its largest cells, in agreement with Toner et al. who found that 20-cell clusters could traverse 5-10 $\mu \mathrm{m}$ constrictions in a microfluidic device following an unfolding process (Au et al., 2016).

The $15 \mu \mathrm{m}$ filters contained the largest diversity of clusters, while no large clusters were found on the following 12, 10 or $8 \mu \mathrm{m}$ filters, suggesting that $15 \mu \mathrm{m}$ filters efficiently capture clusters, and were thus considered optimal. Using $0.1 \mathrm{~mL} \mathrm{~min}^{-1}, 15 \mu \mathrm{m}$ filters yielded $\Delta \mathrm{P}$ of $0.61 \mathrm{~Pa}$ that resulted in a $v_{\max }$ of 
Meunier et al.

$340 \mu \mathrm{m} \mathrm{s}^{-1}$ and a $\tau_{\max }$ of $0.35 \mathrm{~Pa}$, below stress in capillaries (Ballermann et al., 1998) (Tables S3), which provides further support that large clusters can be captured by $\mathrm{G} \mu \mathrm{F}$ without disrupting them.

\section{Release of OV-90 clusters, cluster size distribution and viability}

To characterize cell release from the filter, OV-90 single cells and clusters were first stained in suspension, then spiked in diluted blood. Samples were divided in three and one third was used to determine clusters size distribution before filtration (positive control). The two other samples were filtered at $0.1 \mathrm{~mL} \mathrm{~min}^{-1}$, then cells were released by placing the cartridge upside down and flowing $5 \mathrm{~mL}$ of PBS in the reverse direction, either at 0.1 or $1.0 \mathrm{~mL} \mathrm{~min}^{-1}$, and their size distribution was characterized (Figure 3D).

The average area of clusters before filtration $\left(795 \pm 82 \mu \mathrm{m}^{2}\right)$ and after release at $0.1 \mathrm{~mL} \mathrm{~min}^{-1}(974 \pm 57$ $\mu \mathrm{m}^{2}$ ) was comparable, with clusters from $\sim 100 \mu \mathrm{m}^{2}$ to 5000 and $10000 \mu \mathrm{m}^{2}$, respectively. Following release at $1.0 \mathrm{~mL} \mathrm{~min}^{-1}$, average cluster area decreased to $486 \pm 191 \mu^{2}$, and the cluster distribution shrank with a strong increase in small cluster frequency and a significant loss of larger clusters. Clusters smaller than $400 \mu \mathrm{m}^{2}$ represented $23 \pm 6 \%$ of the total cluster population before filtration, $16 \pm 7 \%$ after release at $0.1 \mathrm{~mL} \mathrm{~min}^{-1}$, and up to $59 \pm 9 \%$ after release at $1 \mathrm{~mL} \mathrm{~min}^{-1}$. Although high flow rates are likely to help dislodging clusters, release efficiency decreased from $\sim 83 \%$ at $0.1 \mathrm{~mL} \mathrm{~min}^{-1}$ to $\sim 60 \%$ at $1.0 \mathrm{~mL} \mathrm{~min}^{-1}$, further highlighting the susceptibility of clusters to shear. At $0.1 \mathrm{~mL} \mathrm{~min}{ }^{-1}, \mathrm{G} \mu \mathrm{F}$ can both capture and release clusters while preserving their integrity.

Cell viability was measured after release from filters using blood samples spiked with 500 OV-90 single cells and clusters (Figure 3E). Dilution, rinsing, and release were performed with complete ovarian surface epithelial (OSE) culture medium, PBS, or fetal bovine serum (FBS) at $4^{\circ} \mathrm{C}$ and $23^{\circ} \mathrm{C}$. A flow rate

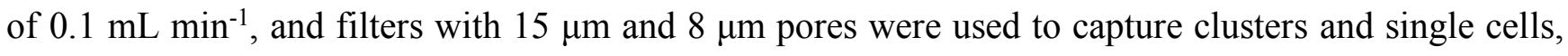
respectively. Single cells viability varied strongly with temperature; optimal conditions being $\mathrm{PBS}$ at $4{ }^{\circ} \mathrm{C}$ $(75.7 \pm 9.1 \%)$. Cluster viability was independent of the tested parameters and stable at $\sim 70-80 \%$, exceeding single cell viability in each case, except when using PBS at $4^{\circ} \mathrm{C}$ which raised single cell viability to the level of that of clusters. The preservation of cell-cell or cell-matrix interactions within clusters could explain their enhanced viability, and the lower effect of temperature compared to single cells. The loss of interaction with the extracellular matrix during shedding from the tumor induces 
Meunier et al.

activation of anoikis (Frisch and Francis, 1994), and low temperature could slow down cell death mechanisms, thus accounting for increased single cell viability. As a positive control, cells were simply incubated for $5 \mathrm{~h}$ at $37^{\circ} \mathrm{C}$ in low-adhesion dishes and the viability was similar to that of cells released after $\mathrm{G} \mu \mathrm{F}$, indicating that neither single cell nor cluster viability were negatively affected by the $\mathrm{G} \mu \mathrm{F}$ process. The full process from capture to release lasts $\sim 5 \mathrm{~h}$, and cluster viability was $\sim 75 \%$, and might be further increased by reducing filtration time by using filters with larger diameters. 
A

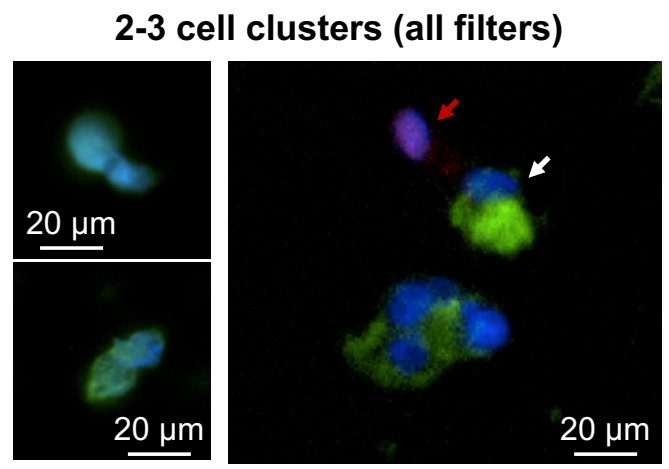

B

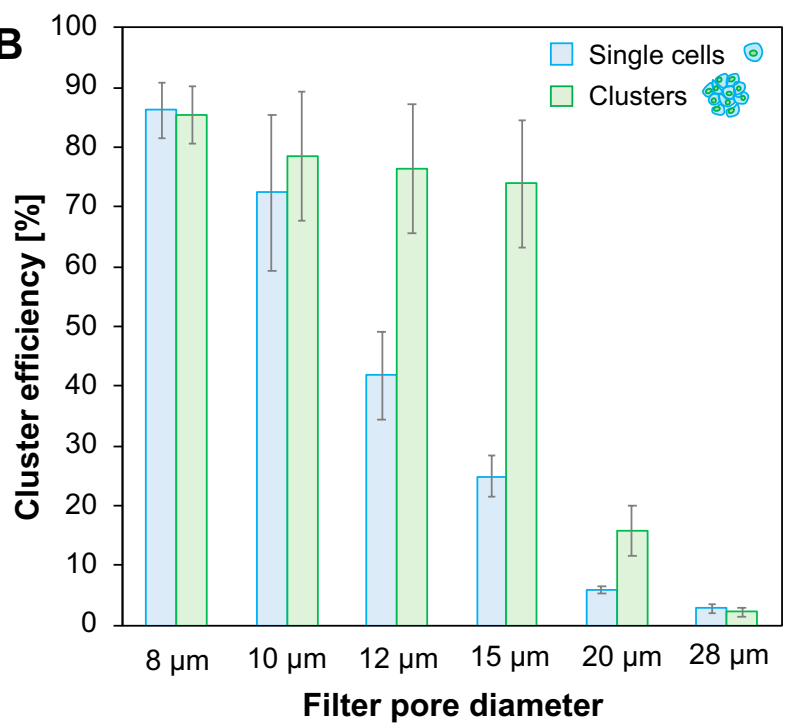

D

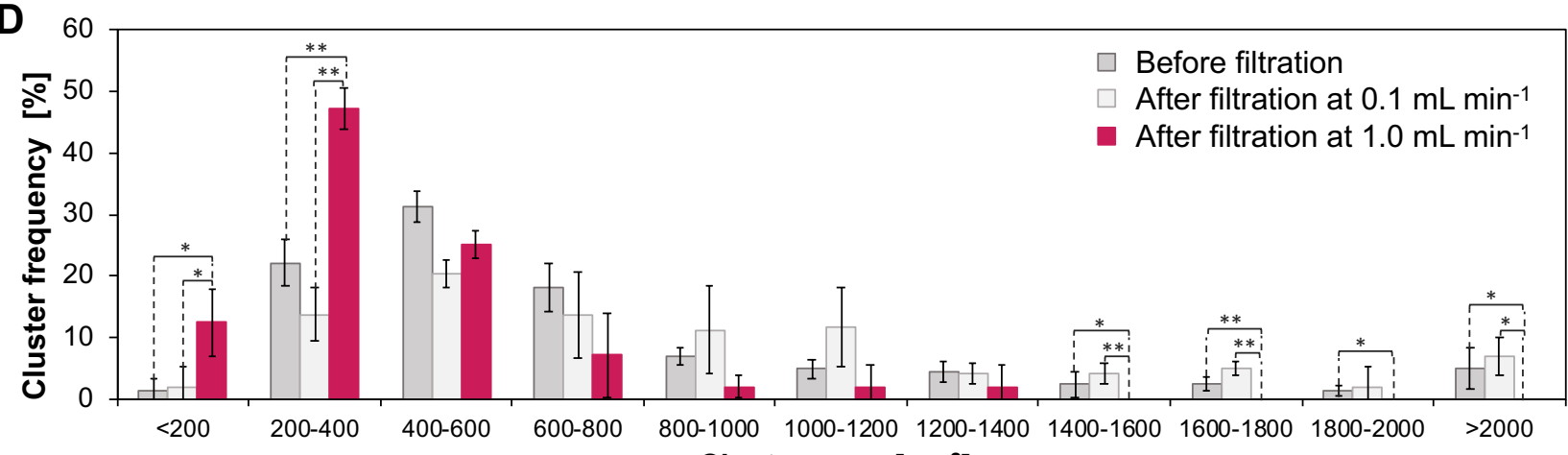

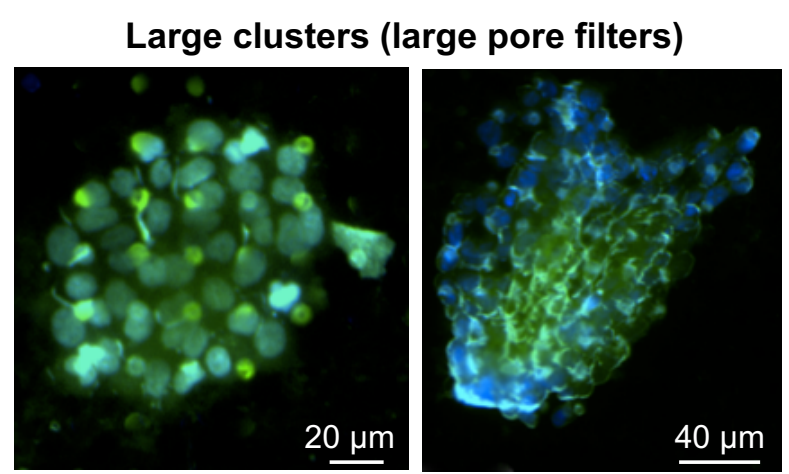

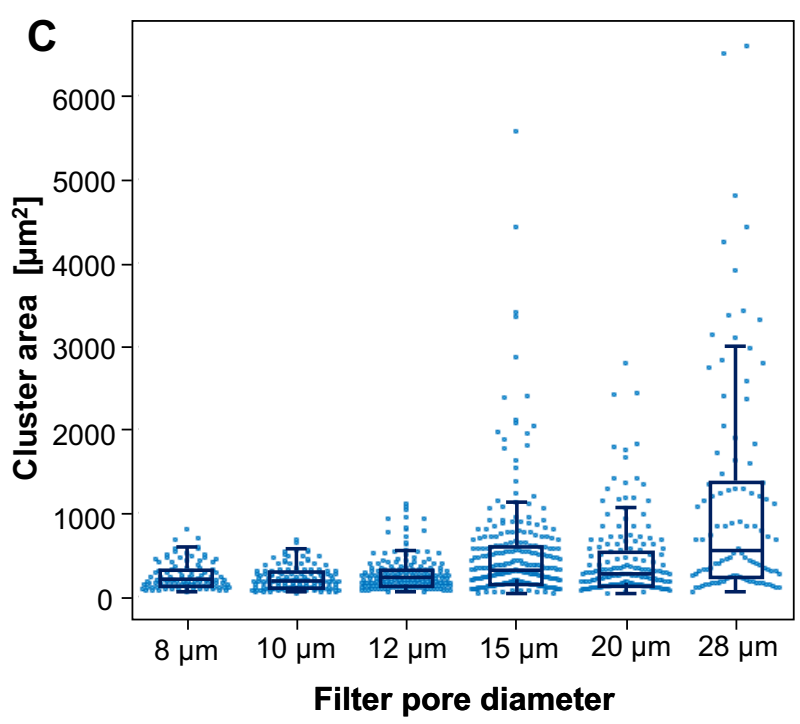

Filter pore diameter

Cluster area $\left[\mu^{2}\right]$
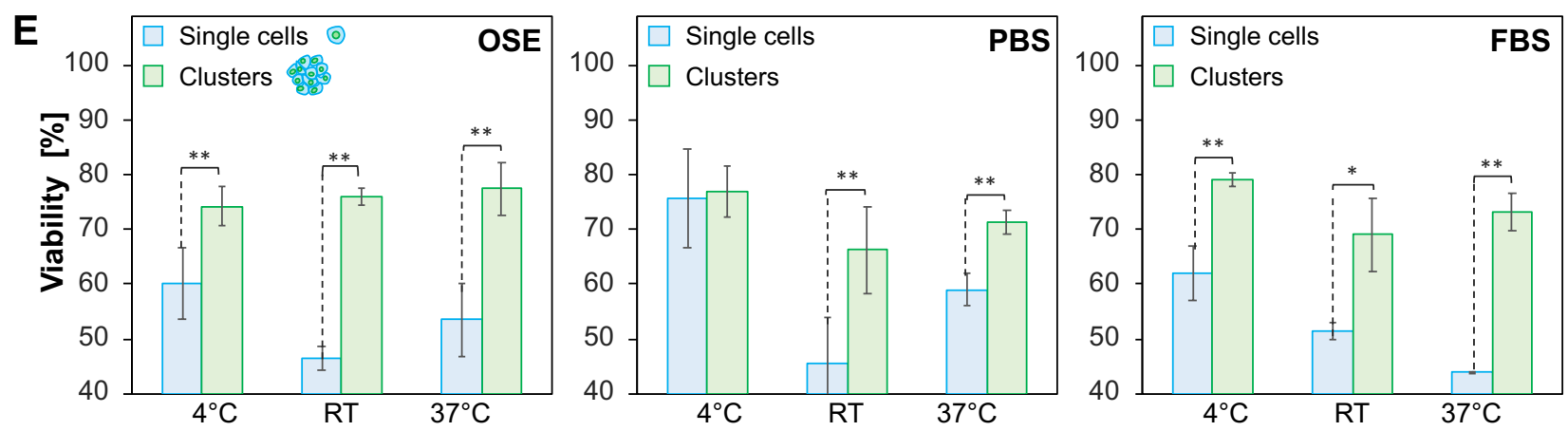
Figure 3. Characterization of clusters captured by $G \mu \mathbf{F}$. (A) Fluorescence images of representative large and small clusters. Cells were stained for CK (green), CD45 (red) and with DAPI (blue). CTC-like cells (white arrow) are $\mathrm{CK}^{+} / \mathrm{CD} 45^{-} / \mathrm{DAPI}^{+}$and $\mathrm{WBCs}$ (red arrow) are $\mathrm{CK}^{-} / \mathrm{CD}^{2} 5^{+} / \mathrm{DAPI}^{+}$. (B) Capture efficiency depending on pore size, measured with OV-90 single cells (blue) and clusters (green) spiked in diluted blood. (C) Scatterplot and box plot of the size distribution of clusters captured by serial $\mathrm{G} \mu \mathrm{F}$ through filters with deceasing pore size $(28,20,15$, $12,10$ and $8 \mu \mathrm{m})$ for three replicated experiments $(\mathrm{n}=145,300$ and 550 spiked clusters). The boxes range from $25^{\text {th }}$ and $75^{\text {th }}$ percentiles, the whiskers correspond to $91^{\text {st }}$ and $9^{\text {th }}$ percentiles, and the horizontal lines represent the medians. (D) Cluster size distribution before filtration (dark grey, $\mathrm{n}=80,39$ and 37) and after filtration at $0.1 \mathrm{ml}$ $\min ^{-1}$ and release by flowing PBS at either at $0.1 \mathrm{~mL} \mathrm{~min}^{-1}$ (light grey, $\mathrm{n}=61,30$ and 35) or $1 \mathrm{~mL} \mathrm{~min}^{-1}(\mathrm{red}, \mathrm{n}=$ 37, 31 and 19). (E) Viablility of single cells (blue) and clusters (green) after filtration, rinsing, and release using complete OSE culture medium, PBS, or FBS at $4^{\circ} \mathrm{C}$, at RT $\left(22-23^{\circ} \mathrm{C}\right)$, and after $5 \mathrm{~h}$ incubation in low adhesion plates (no processsing) at $37^{\circ} \mathrm{C}$. Error bars correspond to the standard deviation of three replicated experiments. $p$ $<0.01$ **; $^{*}<0.05: *$. See also Figure S3.

\section{Capture of circulating tumor cell clusters (cCTCs) from mouse blood}

$\mathrm{G} \mu \mathrm{F}$ was tested with blood from ovarian orthotopic mouse models after injecting OV-90 or OVCAR-3 cells, with high and low invasive potential (Provencher et al., 2000), respectively, and collecting their blood after sacrifice. Orthotopic xenograft results in spontaneous metastasis that closely mimic dissemination from the primary tumor in human cancers (Lengyel et al., 2014), thus recapitulating early events in disease progression. Half of each sample was saved for growth analysis, while the other half was filtered using $8 \mu \mathrm{m}$ filters to capture the gamut of scCTCs and cCTCs. From the blood of three OV90 mice, 505/5, 259/9 and 582/4 scCTCs/cCTCs were captured, thus accounting for $<5 \%$ of the total CTC population, in agreement with published results (Aceto et al., 2014). Cluster with size between 2$100+$ cells were found in every mouse.

Next, blood from a single OVCAR-3-GFP (green fluorescent protein) mouse was diluted and divided into four aliquots. One quarter was saved for further growth analysis, and the three others filtered through $15 \mu \mathrm{m}$ filters at $0.1 \mathrm{~mL} \mathrm{m^{-1 }}$. Respectively 55, 40, and 49 cCTCs were found in each replicate, falling well within the variation expected based on Poisson statistics alone. The size of cCTCs again varied from $2-100+$ cells, their frequency was highest for small clusters, with 2-cell and 3-cell clusters representing $27-33 \%$ and $5-12 \%$ of all cCTC events, respectively, and the larger clusters were rarer, with consistent results across the triplicates (Figure $4 \mathrm{~A}$ and $4 \mathrm{~B}$ ). cCTCs as big as $30000-35000 \mu \mathrm{m}^{2}\left(\varnothing_{\mathrm{eq}} \sim 0.6 \mathrm{~mm}\right)$ were captured directly from the mouse's bloodstream, consistent with previous observations of large cCTC 
aggregates in blood (Au et al., 2016). These results give us high confidence in the reproducibility and performance of $\mathrm{G} \mu \mathrm{F}$ to isolate cCTCs spontaneously formed in vivo.

The blood samples of OV-90 and OVCAR-3-GFP saved earlier were filtered through $15 \mu \mathrm{m}$ filters, and the cCTCs were released and cultured in regular and low-adhesion culture flasks at $37^{\circ} \mathrm{C}$. Both OV-90 and OVCAR-3-GFP cCTCs adhered and spread after a few hours in adherent flasks forming confluent layers after 7-8 days. Migration assays revealed similar growth behaviors for both cell lines, where a full coverage of the cell-free area was reached after 6 days (Figure S4A-D). In low adhesion flasks, OV-90 and OVCAR-3-GFP cCTCs growth also occurred in suspension. After 7 days of incubation, the cluster average diameter increased from $1500-2000 \mu \mathrm{m}^{2}\left(\varnothing_{\mathrm{eq}} \sim 40-50 \mu \mathrm{m}\right)$ at day zero for both cell lines, to $\sim 6000$ and $\sim 15500 \mu \mathrm{m}^{2}$ for OVCAR-3-GFP and OV-90, respectively (Figure 4C). Both cell lines exhibited a broad cluster size distribution (Figure S4E-G) with cCTCs from 160 to more than $20000 \mu m^{2}$; OV-90 clusters were larger than OVCAR-3-GFP however, in agreement with a higher invasive phenotype. After day 2, OV-90 clusters kept increasing in size while OVCAR-3-GFP plateaued and at the same time a significant number of dying OVCAR-3-GFP was observed, likely due to a higher sensitivity of OVCAR3 clusters to hypoxic conditions in the core.

\section{cCTCs can help understand dissemination in ovarian cancer}

In EOC, Snail, a zinc finger transcription repressor, can activate zinc finger E-box-binding homeobox 1 (ZEB-1) that induce the loss of adhesion proteins such as E-cadherin (E-Cad) (Cano et al., 2000) and zonula occludens-1 (ZO-1) (Polette et al., 2007). As in many cancers, cell adhesion plays a critical role in the dissemination of EOC, and notably, the loss of E-cad and ZO-1 (Polette et al., 2007) was correlated with disease aggressiveness by promoting tumor growth, invasiveness, and resistance to chemotherapy (Haslehurst et al., 2012, Wang et al., 2015). cCTCs were isolated from the blood of two OV-90 and two OVCAR-3 mice, as well as from the ascites of one of the OVCAR-3 mice. After release, cCTCs were stained for aggressiveness markers: E-Cad, ZO-1, ZEB-1 and Snail (Figure 4D and 4E). cCTCs from ascites and blood had not yet been compared to the best of our knowledge, and interestingly, different phenotypes were dominant in each sample. OV-90 cCTCs from blood strongly expressed ZO-1, ZEB-1 and Snail, but exhibited extremely low levels of E-Cad. OVCAR-3 cCTCs captured from blood also displayed a weak expression of E-cad but lower expression of ZO-1, ZEB-1 and Snail, consistent with a less aggressive behavior. OVCAR-3 cCTCs from ascites exhibited significantly lower levels of ZO-1, ZEB-1 and Snail than OV-90 cells from blood, but in the same range or slightly higher than OVCAR-3 
cCTCs from blood of the same mouse. In all samples, cCTCs were strongly heterogeneous with variable levels of epithelial markers. Additional staining for the mesenchymal marker vimentin (Vim) (Pantel and Speicher, 2016), showed that blood OV-90 cCTCs exhibited a pronounced mesenchymal phenotype with low E-cad and high Vim levels (Figure 4F). Blood and ascites OVCAR-3 cCTCs displayed a hybrid epithelial/mesenchymal (E/M) phenotype, each individual cell co-expressing epithelial and mesenchymal markers. These results support the contribution of EMT in cCTCs dissemination and confirm the potential of $\mathrm{G} \mu \mathrm{F}$ for molecular and functional analysis of living cCTCs. 
A
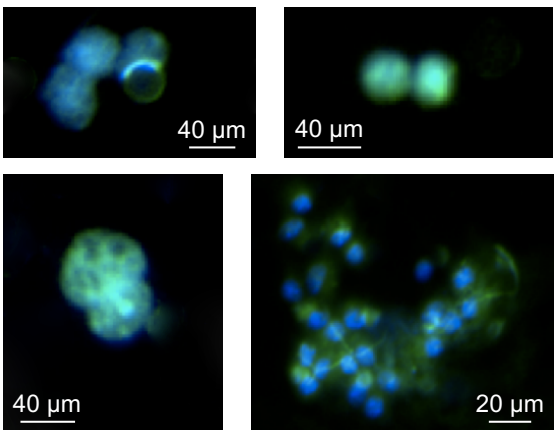

B

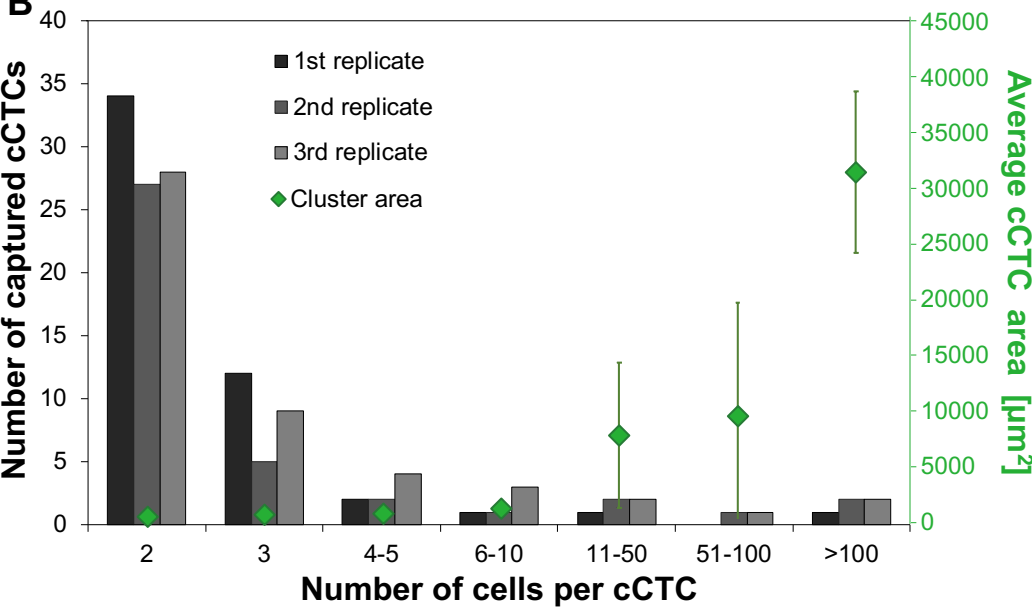

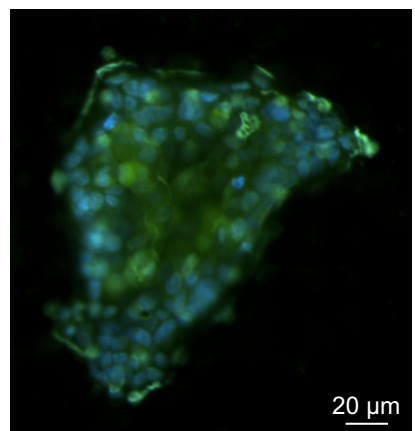

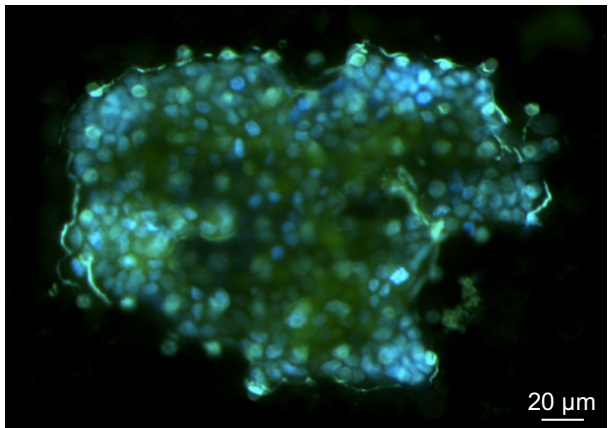

C

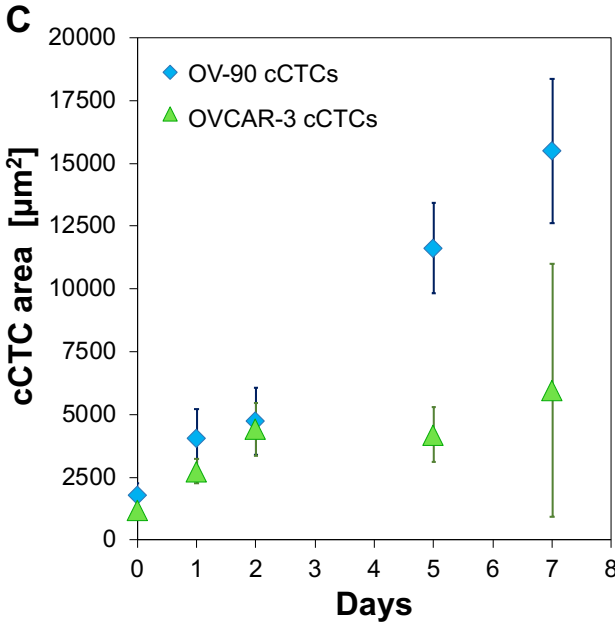

D OV-90 from blood
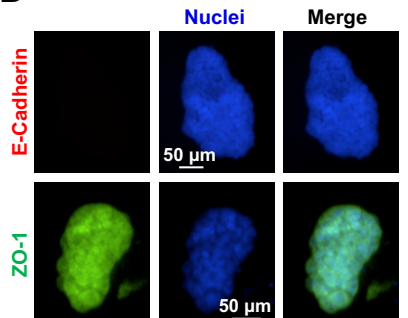

$50 \mu \mathrm{m}$
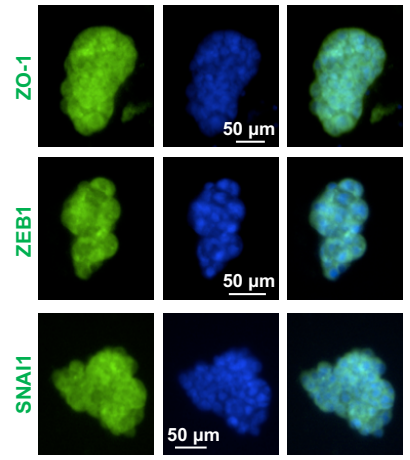

$\mathbf{F}$

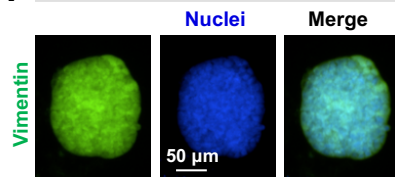

OVCAR-3 from blood
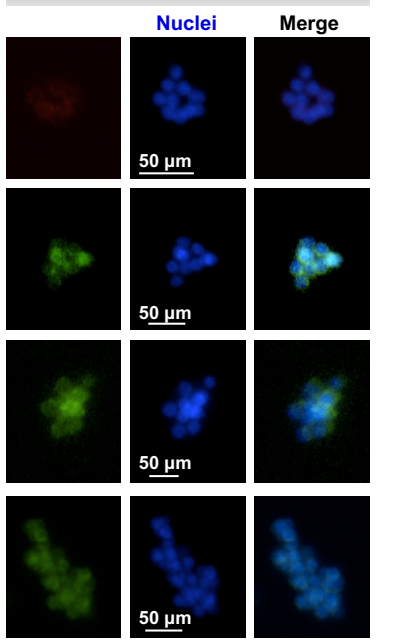

OVCAR-3 from blood

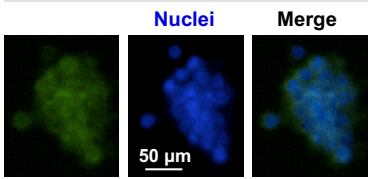

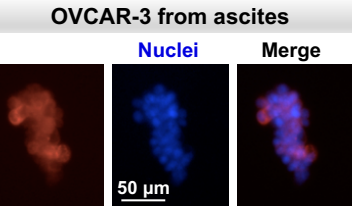
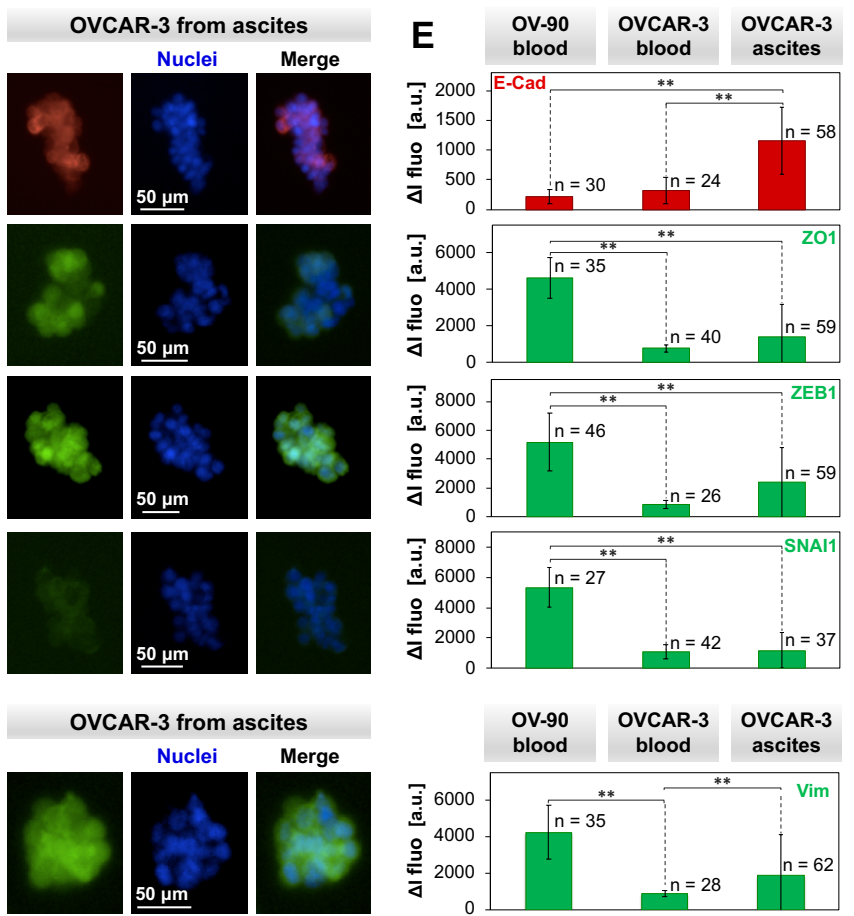

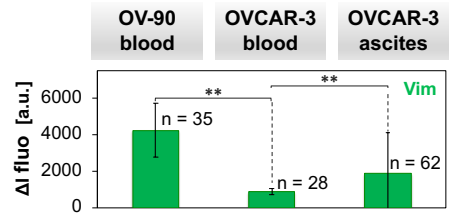

Figure 4. cCTCs captured from mouse. A. Examples of fluorescence images of OVCAR-3-GFP cCTCs captured from mouse blood. Nuclei were stained with DAPI. B. Size distribution of the OVCAR-3-GFP cCTCs captured in three replicates from a single mouse. cCTC area is averaged over three replicates. C. Evolution of the average area 
of OV-90 and OVCAR-3-GFP cCTCs overtime during incubation in low adhesion culture flasks. Error bars correspond to the standard deviation of three replicated experiments. D. Fluorescence images of OV-90 cCTCs captured from mice blood, and OVCAR-3 cCTCs from the blood and ascites of the same mouse. cCTCs were stained for aggressiveness markers E-Cad, ZO-1, ZEB1 and Snail. Nuclei were stained with DAPI. E. Expression levels of E-cad, ZO-1, ZEB-1 and Snail in OV-90 and OVCAR-3 cCTCs from blood or ascites. F. Additional staining for mesenchymal Vim. OVCAR-3 cCTCs from blood and ascites exhibit a hybrid E/M phenotype. Error bars correspond to the standard deviation between cCTCs. See also Figure S4.

\section{Capture of cCTCs from epithelial ovarian cancer (EOC) patients}

$\mathrm{G} \mu \mathrm{F}$ was used for isolating CTCs from the blood of 10 EOC patients (Table 1). A single blood sample was collected from 5 patients with high-grade serous carcinoma (HGSC), all at high stage (metastatic), before (P1) or after several cycles of chemotherapy (P2-P5). A single blood sample was also drawn from a second group of 5 patients with other histological subtypes of EOC, at lower stage, and all before chemotherapy (P6-P10). 
Table 1. Patient information. The time at which blood was drawn and analyzed as well as the CA125 concentration in blood at the time of CTC analysis is provided.

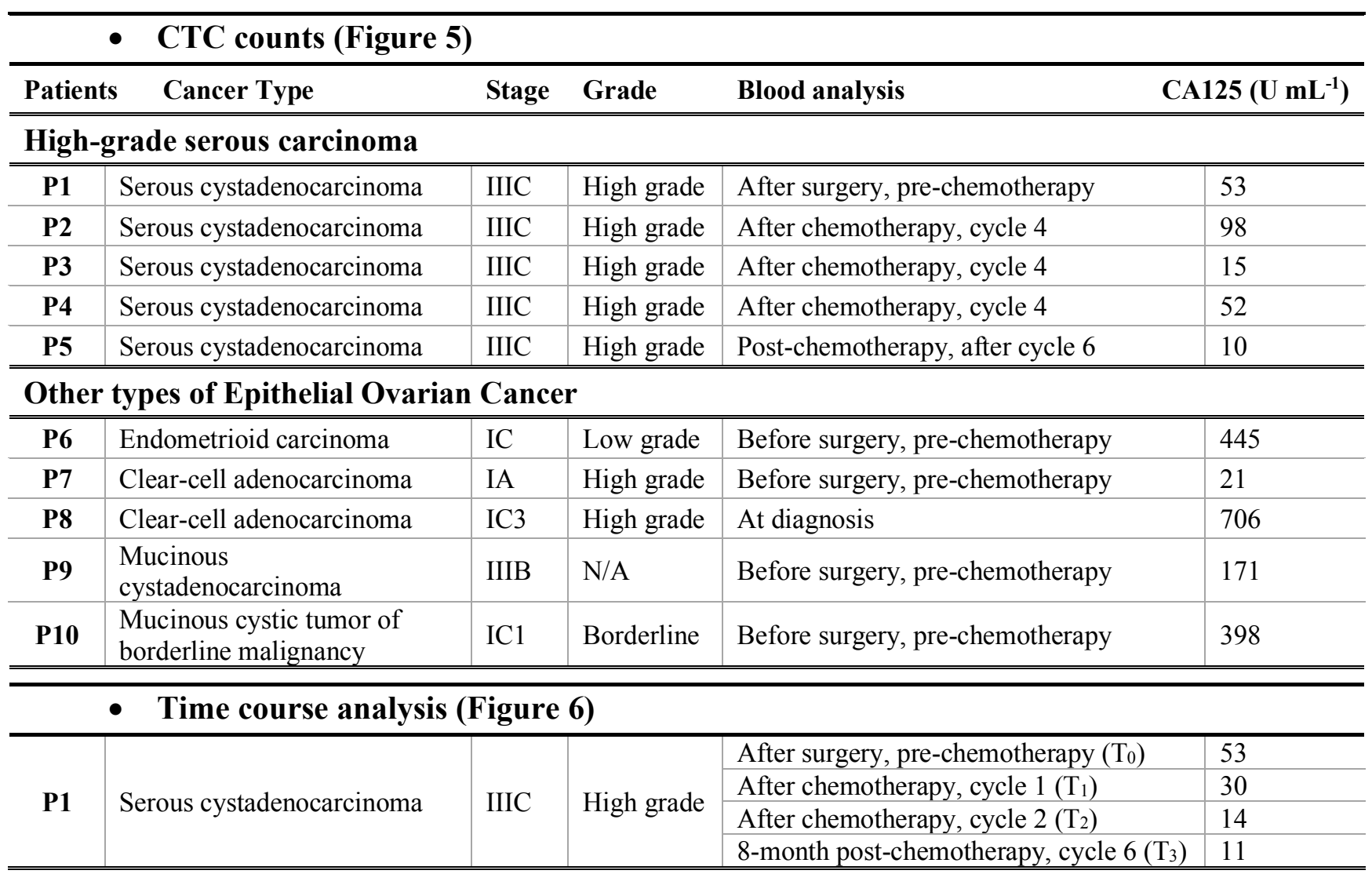

The 10 blood samples (P1-P10) were processed by filtering first with $15 \mu \mathrm{m}$ followed by $8 \mu \mathrm{m}$ filters to capture CTCs. scCTCs and cCTCs were found in every sample, with different relative frequency for each patient. For patients with HGSC (Figure 5A), the number of scCTCs per mL of blood varied from 22 (P4) to 145 (P1), and the number of cCTCs from 1 (P4) to 62 (P1). For P1 (before chemotherapy), cCTCs represented up to $\sim 30 \%$ of all CTCs capture events. For patients with HGSC, with good response after several cycles of chemotherapy, as indicated by low CA125 levels (Table 1), the proportion of cCTCs events was lower, varying from $\sim 4 \%$ (P2) to $\sim 8 \%$ (P5). For patients with other types of EOC at lower stage (Figure 5B), the number of scCTCs per $\mathrm{mL}$ of blood varied from 25 (P9) to 155 (P7), and the number of cCTCs from 3 (P9) to 45 (P6), and cCTCs represented from $\sim 7 \%$ (P10) to $30 \%$ (P6) of all CTC events.

To better understand the relative contribution of cCTCs and ScCTCs, the number of cells captured as cCTCs was quantified by counting the number of cells in each cluster. The high transparency and low 
autofluorescence of the filters allowed us to precisely determine the number of cells for small clusters and estimate it for large clusters. For patients with metastatic HGSC (Figure 5C), cells in clusters represented $\sim 71 \%$ of all CTCs for P1, and was significantly lower for patients having followed several cycles of chemotherapy, varying from $\sim 12 \%$ (P2) to $\sim 17 \%$ (P5). For patients with low stage EOC (Figure $5 \mathrm{D}$ ) and no chemotherapy, the proportion of cells in clusters varied from $\sim 14 \%$ (P10) to $\sim 82 \%$ (P6). Overall, in $8 / 10$ patients, cells in clusters corresponded to $\sim 10-25 \%$ of all CTCs, and represented the majority of CTCs in 2/10 patients, which is significant given their high metastatic potential. Collectively, these results reveal an unexpected prevalence of cCTCs in patients with various histological subtypes of ovarian cancers, at both high and low stage.

For patients with metastatic HGSC, the size distribution of cCTCs varied from 2-50 cells (Figure 5E). In patients responding to therapy, small cCTCs with 2 cells were the most frequent, corresponding to 50$90 \%$ of all cCTC events; clusters with 3-4 cells were found in $4 / 4$ patients (10-50\% of all cCTC events); and clusters with more than 11 cells were only found in P2. In P1 (before chemotherapy), the proportion of small clusters was lower, with 2- and 3-cells clusters representing 36\% of all cCTCs events together, while clusters with more than 4 cells represented the majority (64\%). In patients with other EOC at lower stage and no chemotherapy, the size distribution of cCTCs varied from 2-100+ cells (Figure 5F), mirroring the one observed in mice. For P6, with endometrioid carcinoma, 2- and 3-cell clusters represented $28 \%$ and $15 \%$ of all cCTCs, respectively; clusters with more than 4 cells represented $57 \%$, including clusters with more than 50 and 100 cells. For P7 and P8, with clear-cell adenocarcinoma, and for P9 and P10 with mucinous disease, small clusters (2-3 cells) were the most frequent, representing 89$100 \%$ of all cCTCs events; no cluster larger than 3 cells were found in P10 (borderline), and cluster larger than 5 cells were only found in P7 and represented only $2 \%$ of all cCTCs. 
Advanced high-grade serous carcinoma

A

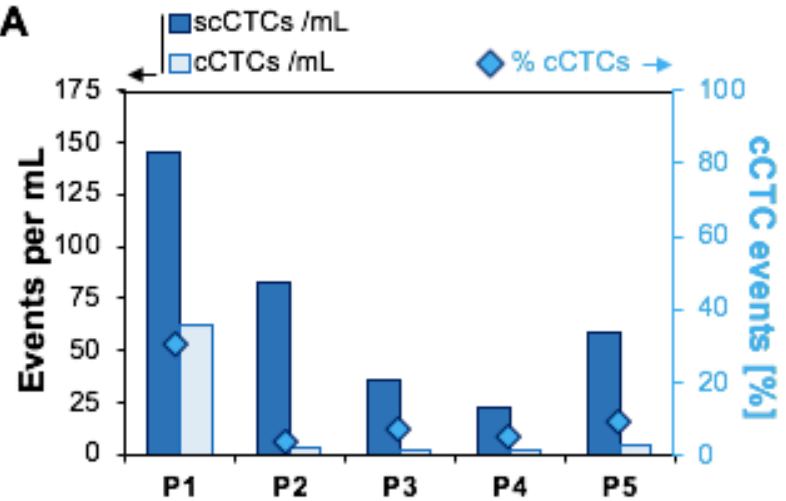

C

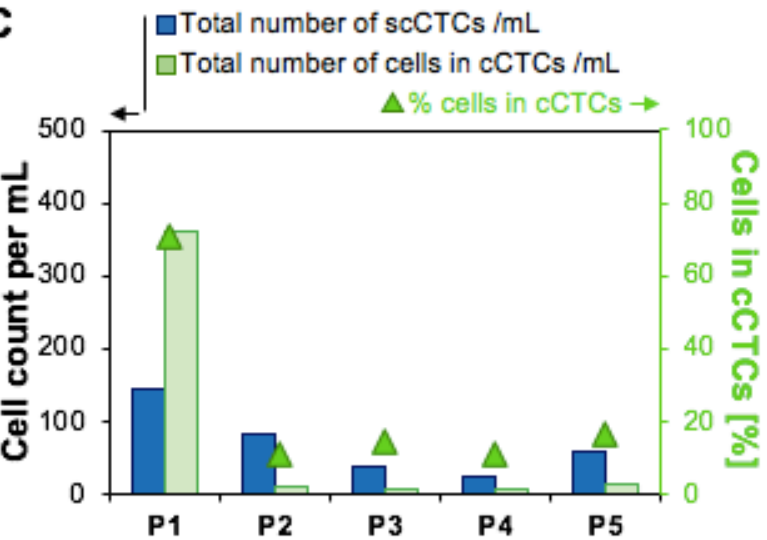

E

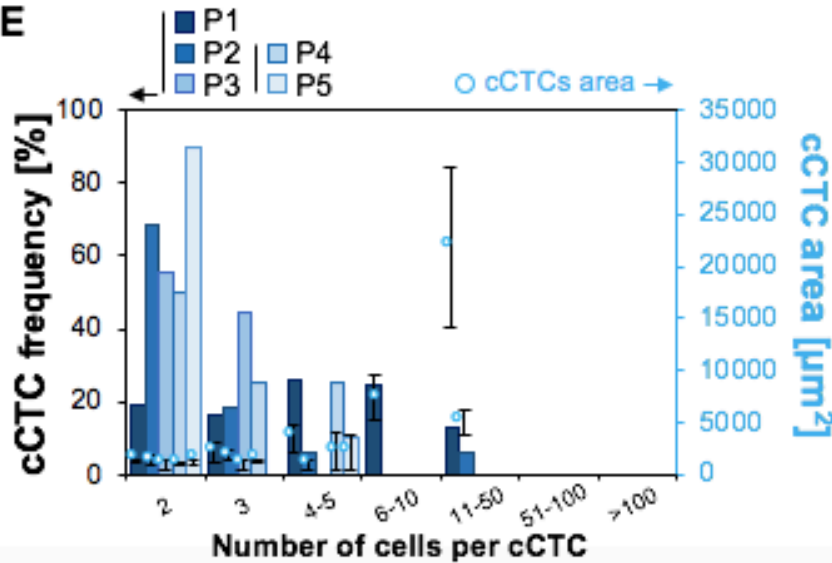

Other types of EOC at lower stage
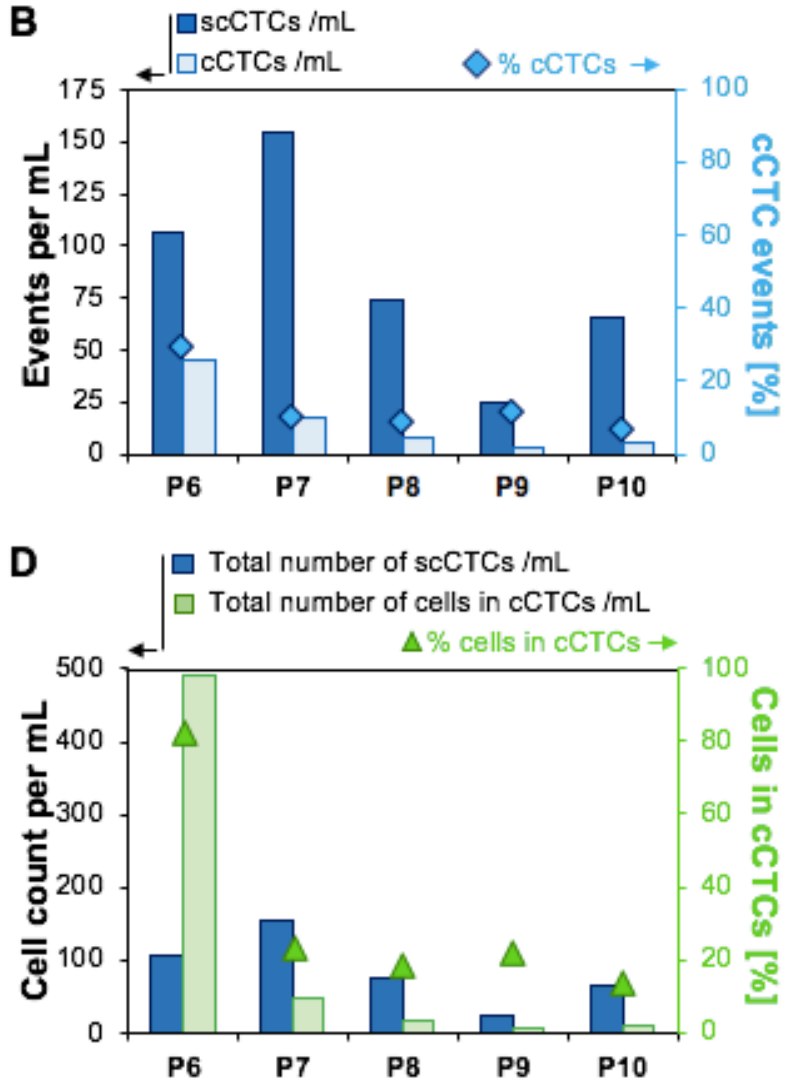

$\mathbf{F}$

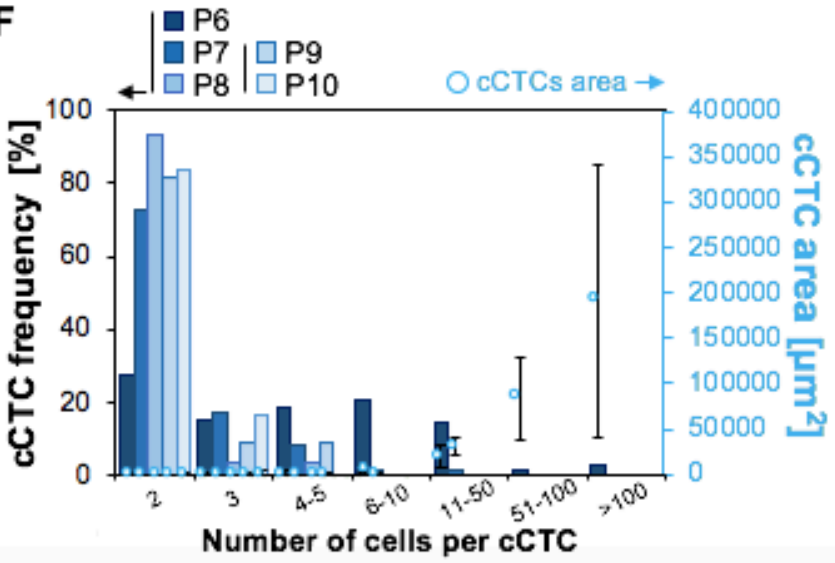

Figure 5. Count and morphological characterization of CTCs captured from the blood of EOC patients with advanced high-grade serous carcinoma (left) and other types EOC at lower stage (right). (A-B) Concentration of scCTCs and cCTCs in the blood of patients P1 to 10. The diamonds represent the frequency of cCTC events. (C-D) Number of scCTCs and of cells in cCTCs per milliliter of blood, in each patient sample. The triangles correspond to the percentage of cells in cCTCs vs. all CTCs. (E-F) Size distribution of cCTCs captured from the blood of P1 to P10. The area covered by cCTC clusters is provided (dots) on an indicative basis. Error bars correspond to the standard deviation between cCTC of same number of cells in each patient. 


\section{Chemotherapy differentially reduces scCTC and cCTC burden}

Finally, a time-course study was conducted for P1 by collecting blood sample during the course of chemotherapy (Figure 6A). The concentration of CA125 in blood was used to track response to therapy and levels below the cut-off at $35 \mathrm{U} \mathrm{mL}^{-1}$ indicate a good response. The CA125 level of P1 was reduced from $>2000 \mathrm{U} \mathrm{mL}^{-1}$ at diagnosis to $\sim 50 \mathrm{U} \mathrm{mL}^{-1}$ after surgery and further decreased over the first three cycles of chemotherapy, plateauing at $\sim 10 \mathrm{U} \mathrm{mL}^{-1}$. CTCs analysis was performed at four times points: after surgery and before chemotherapy $\left(\mathrm{T}_{0}\right)$, after the first cycle of chemotherapy $\left(\mathrm{T}_{1}\right)$, a few days before the third cycle of chemotherapy $\left(T_{2}\right)$, and post treatment $\left(T_{3}\right)$; no pre-surgery samples were available for CTC isolation (Table 1). The number of scCTCs and cCTCs was stable between $\mathrm{T}_{0}$ and $\mathrm{T}_{1}$, with $\sim 140$ scCTCs and $\sim 60$ cCTCs per milliliter. Then, both scCTCs and cCTCs decreased in number as therapy progressed, but followed different trajectories. The number of scCTCs initially remained similar until the third cycle of chemotherapy, with $132 \mathrm{scCTCs}$ per $\mathrm{mL}$ at $\mathrm{T}_{2}$, then decreased to 11 after 6 cycles of chemotherapy $\left(\mathrm{T}_{3}\right)$. The number of cCTCs decreased from 62 to 2 in a gradual manner. Thus, while the CA125 level stopped changing after 3 cycles, the number of scCTCs and cCTCs continued dropping.

Cluster size distribution was determined at the four time points (Figure 6B). Before chemotherapy $\left(\mathrm{T}_{0}\right)$ and after the first cycle $\left(\mathrm{T}_{1}\right)$, cCTC size distribution was similar, respectively with $35 \%$ and $40 \%$ of cCTCs with $2-3$ cells, $52 \%$ and $38 \%$ of cCTCs with $4-10$ cells, $12 \%$ and $15 \%$ of cCTCs with $11-50$ cells. No cCTCs larger than 50 cells were captured at $\mathrm{T}_{0}$, and, at $\mathrm{T}_{1}$, clusters with $50-100+$ cells corresponded to $\sim 4 \%$ of all cCTCs. This small increase in the number of large clusters between $\mathrm{T}_{0}$ and $\mathrm{T}_{1}$ is likely due to sampling variability as small blood volume were processed $(3-4 \mathrm{~mL})$, and larger clinical study would help determine its significance. Then, after the second cycle of chemotherapy $\left(\mathrm{T}_{2}\right)$, in addition to the strong reduction in the number of $\mathrm{cCTCs}$, the $\mathrm{cCTCs}$ size was significantly reduced, with the majority ( $\sim 94 \%)$ of cCTCs with $2-5$ cells and only 1 cCTCs with more than 11 cells. Finally, post-treatment $\left(\mathrm{T}_{3}\right)$, only 2- and 3-cells clusters were captured. After the second cycle of chemotherapy $\left(\mathrm{T}_{2}\right)$ and, to a much greater extent after the last cycle $\left(\mathrm{T}_{3}\right)$, membrane blebbing and leakage of the cell contents was observed suggesting increased death of the cCTCs (Figure 6C). Together, these results indicate that chemotherapy may affect both the number and the size of cCTCs. 


\section{A Patient 1}

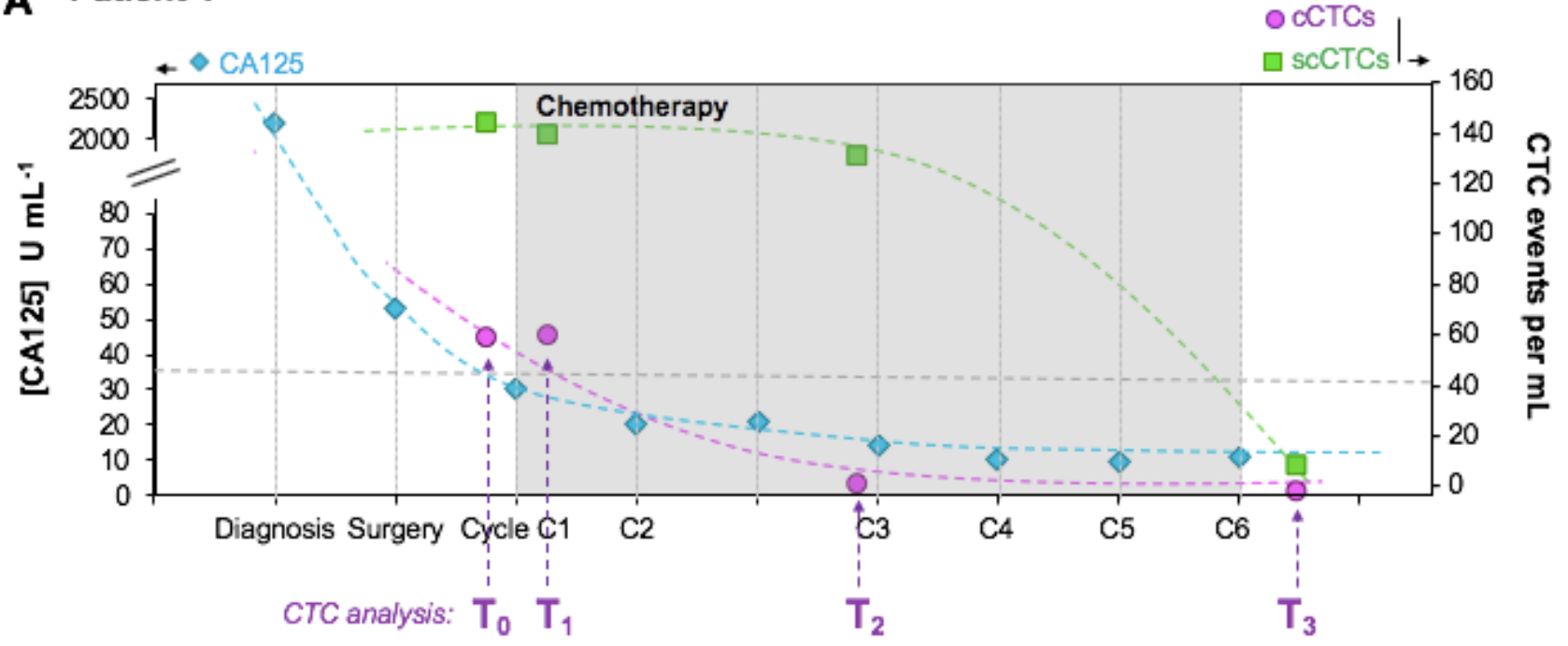

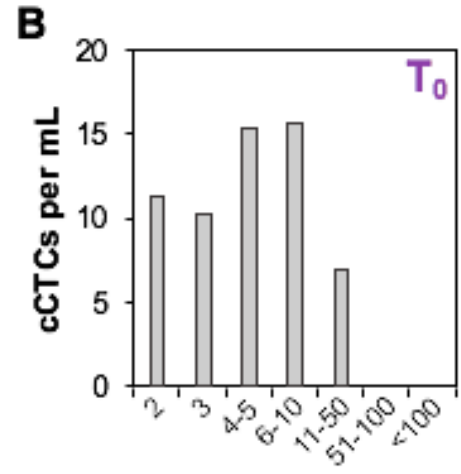

Cells per cCTCs

C

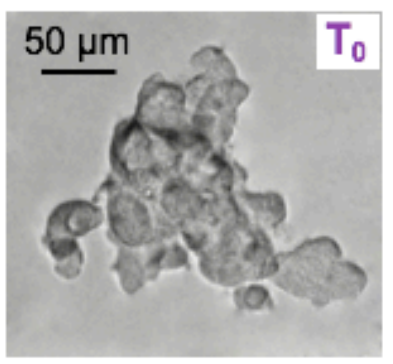

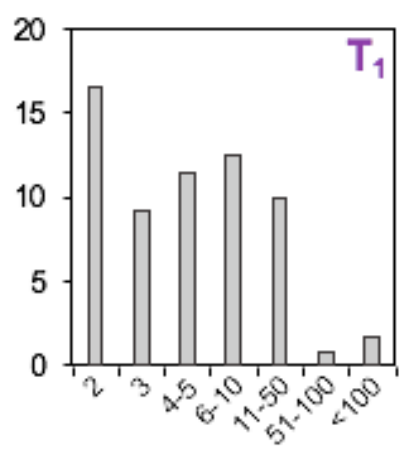

Cells per cCTCs

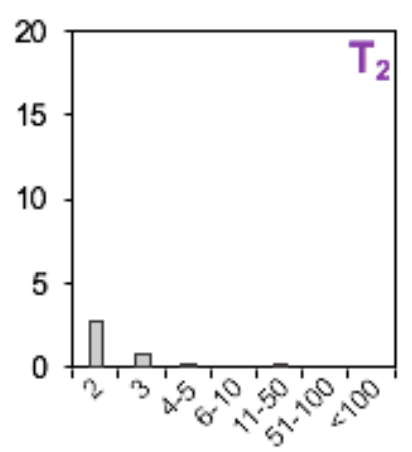

Cells per cCTCs

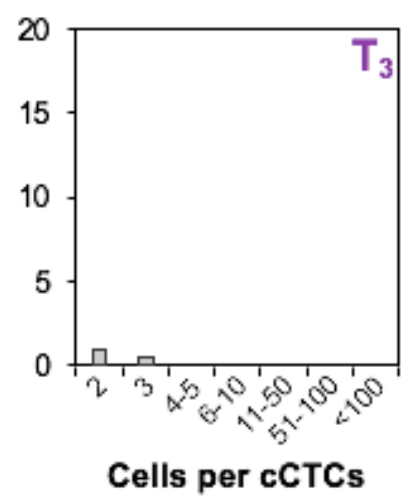

Cells per cCTCs

Figure 6. Time-course study for one metastatic HGSC patient. A. Time course study for P1 who responded to therapy, showing the CA125 concentration as well as the number of scCTC and $\mathrm{CCTC}$ events per $\mathrm{mL}$ of blood at different time points during chemotherapy. B. cCTCs size distribution and C. representative bright field images of cCTCs captured from the blood of P1 at four time points during follow-up. 


\section{Discussion}

$\mathrm{G} \mu \mathrm{F}$ using filters with $8 \mathrm{~mm}$ diameter and $15 \mu \mathrm{m}$ pores (20\% porosity) for enriching cCTCs, and $8 \mu \mathrm{m}$ pores ( $8 \%$ porosity) for capturing the cCTCs that slipped through the large filters and scCTCs, is an effective method for capturing the compendium of CTCs, and is uniquely suited for capturing fragile clusters. Microfiltration was among the very first methods used to capture rare CTCs (Fleischer et al., 1964, Seal, 1964), and whereas several applications for scCTC isolation were successfully developed (Adams et al., 2014, Tang et al., 2014, Lim et al., 2012), its use for cCTCs had not been widely explored. Sarioglu et al. recently tested cCTCs isolation with low porosity $(<5 \%)$ (Fleischer et al., 1964, Holdich et al., 2006) track-etched membranes with $5 \mu \mathrm{m}$ pores, but recovered only $\sim 36 \%$ of spiked cCTCs at 0.07 $\mathrm{mL} \min ^{-1}$ (Sarioglu et al., 2015). Here, the adoption of higher porosity filters, gravity (pressure) driven flow, larger pores, and in-depth optimization were key to the high capture yield of cCTCs.

$\mathrm{G} \mu \mathrm{F}$ with $15 \mu \mathrm{m}$ filters and a nominal flow rate of $0.1 \mathrm{~mL} \mathrm{~min}^{-1}$ were found to be optimal for filtration, corresponding to a $\Delta \mathrm{P}$ of $0.61 \mathrm{~Pa}$, a $v_{\max }$ of $340 \mu \mathrm{m} \mathrm{s}^{-1}$, and a $\tau$ of $0.35 \mathrm{~Pa}$ when using buffer, below the velocity (0.5-1.5 $\mathrm{mm} \mathrm{s}^{-1}$ ) (Arfors et al., 1975) and shear (0.5-2 Pa) (Ballermann et al., 1998) measured in blood capillaries. The comparison of $\mathrm{G} \mu \mathrm{F}$ with constant-flow rate filtration through $8 \mu \mathrm{m}$ filters using the same nominal flow rate of $0.1 \mathrm{~mL} \mathrm{~min}^{-1}$ showed higher cluster recovery rate with $\mathrm{G} \mu \mathrm{F}$, that yielded $\sim 80 \%$ vs. only $\sim 30 \%$ with constant-flow rate filtration (Figure 2 ), which was unexpected. To further understand our system, we determined the number of single cells and clusters and assessed the size distribution of OV-90 clusters spiked in blood, before and after filtration, which had not been studied previously to our knowledge. When the flow rate was $0.5 \mathrm{~mL} \mathrm{~min}^{-1}$, this analysis revealed twice as many single cells after filtration than were initially spiked into the sample, which we attributed to shear-driven cluster breakup. The cellular context bears significance in most cancers, and the shear forces in many commonly used isolation techniques are high enough to disrupt cCTCs. Hence, the analysis of the cluster number and size before and after filtration is advisable to assess the impact of a particular filtration method on cCTCs and on scCTCs. During detachment from $15 \mu \mathrm{m}$ filters using flow reversal, higher

flow rates increased the number of small clusters but reduced the apparent cluster release efficiency ( $\sim 60 \%$ at $1.0 \mathrm{~mL} \mathrm{~min}^{-1}$ vs. $\sim 85 \%$ at $0.1 \mathrm{~mL} \mathrm{~min}^{-1}$ ), which again can be attributed to cluster break-up due to shear. A flow rate of $0.1 \mathrm{~mL} \mathrm{~min}{ }^{-1}$ was thus deemed optimal for both capture and release of clusters with our setup.

The cluster capture efficiency for filters with pore sizes from 28 to $8 \mu \mathrm{m}$ was characterized by sequentially filtering the same blood sample spiked with OV-90 clusters through filters with decreasing 
pore size and was found higher for pore sizes of 8-15 $\mu \mathrm{m}$ (Figure 3). The deformability and the large size distribution of both single cells and clusters prevents efficient sorting. For example, small clusters squeezed through pores smaller than their nominal size, consistent with results by Toner and colleagues (Au et al., 2016). With $15 \mu \mathrm{m}$ filters, we recovered 75\% of all clusters from blood, and $\sim 85 \%$ of captured clusters could be released. Remarkably, the size distribution was determined to be the same before and after filtration.

To evaluate the potential for $\mathrm{G} \mu \mathrm{F}$ for clinical use, $2.5-4 \mathrm{~mL}$ of blood from 5 advanced stage (metastatic) and 5 early stage EOC patients were processed using $15 \mu \mathrm{m}$ then $8 \mu \mathrm{m}$ filters, allowing $>85 \%$ recovery of both scCTCs and cCTCs. Whereas scCTCs are commonly isolated, the capture of cCTCs remains a rare occurrence, and were previously isolated from patients with metastatic cancer, but only observed in $5-40 \%$ of the samples. For example a study using the herringbone-chip (HB-Chip) identified cCTCs in 3/19 patients with metastatic prostate or lung cancer (Stott et al., 2010). Using Dean Flow Fractionation, Hou et al. found cCTCs in 1/20 patients with metastatic lung cancer (Hou et al., 2013). Wang et al. found cCTCs in 21/107 patients with metastatic breast cancer at baseline and in 19/77 patients at the first follow-up visit (Wang et al., 2017). Using the Cluster-Chip, cCTCs were found in 30-40\% of patients with metastatic breast, skin or prostate cancer, which constitutes the highest reported yield to date (Sarioglu et al., 2015). In our study using G $\mu \mathrm{F}$, both scCTCs and cCTCs were isolated from every EOC patient, which included patients with metastatic, localized or borderline disease. These results underline the effectiveness of $\mathrm{G} \mu \mathrm{F}$, and considering that cCTCs were typically only detected in a subset of metastatic patients, the question arose whether $\mathrm{G} \mu \mathrm{F}$ is more effective at capturing cCTCs, or whether cCTCs are more prevalent in EOC than in other cancers. Towards resolving this question, samples from three castrate-resistant prostate and one kidney cancer patients, all metastatic, were processed by $\mathrm{G} \mu \mathrm{F}$. cCTCs were again found in every sample (as well as in the urine from the kidney cancer patient, results not shown). Taken together, these results suggest that (i) $\mathrm{G} \mu \mathrm{F}$ is able to isolate cCTCs with high sensitivity, (ii) the current estimates of cCTC (and possibly of scCTCs) constitute a lower bound that is biased by technological limitations, and that (iii) cCTC may in fact be widely prevalent in a number of non-metastatic and metastatic cancers supported by their presence in 10/10 EOC patients at various stages, histological subtypes and in other cancer samples.

G $\mu \mathrm{F}$ using both $15 \mu \mathrm{m}$ and $8 \mu \mathrm{m}$ filters allowed to capture a broad range of CTCs from the blood of EOC patients. cCTCs from 2-100+cells were captured, and small clusters, with 2-3 cells, were the most prevalent, representing from $\sim 35 \%$ to $\sim 95 \%$ of all clusters captured. Clusters occupying $350,000 \mu \mathrm{m}^{2}$ on 
the microfilters, corresponding to $\sim 400$ pores on a $15 \mu \mathrm{m}$ filter, were captured from the blood of EOC patients (and mouse models), indicating that cCTCs can reach large sizes, consistent with other studies (Lecharpentier et al., 2011, Pantel and Speicher, 2016, Yu et al., 2013). cCTCs across a wider range were captured by $\mathrm{G} \mu \mathrm{F}$ and with a higher yield than was previously reported. Indeed, cCTCs with 4-12 cells were captured with the HB-Chip (Stott et al., 2010); 99\% recovery for MDA-MB-231 clusters with more than 4 cells, but only $41 \%$ for 2-cell clusters were reported for the Cluster-Chip (Sarioglu et al., 2015); the ${ }^{\text {neg }}$ CTC-iChip was described as favoring the capture of small clusters and cCTCs with 2-6 cells were isolated from metastatic breast and prostate cancer patients (Ozkumur et al., 2013). Aceto et al. established the metastatic potential of cCTCs being 23- to 50-time the one of scCTCs based on the ratio of scCTCs $v s$. cCTCs captured using the HB-Chip from mouse blood. However, the HB-Chip appears less effective than $\mathrm{G} \mu \mathrm{F}$ or the Cluster-chip for capturing cCTCs. Hence the number of cCTCs may have been underestimated and by extension the metastatic potential of cCTCs, overestimated.

The systematic analysis of the number of cells per clusters captured from EOC patients allowed us to account for the total number of CTCs circulating as cCTCs vs. scCTCs. Typically, $\sim 10-25 \%$ of CTCs were circulating as cCTCs, but in $2 / 10$ patients $\sim 70-80 \%$ of all CTCs were in the form of cCTCs. These results challenge two widely held notions, namely (i) that scCTCs represent the great majority of CTCs, and that (ii) cCTCs are rare considering in two patients they covered the majority of cancer cells in circulation and were found with a frequency of $\sim 45-60 \mathrm{cCTCs} \mathrm{mL}^{-1}$. Additional studies with will be needed to confirm our initial results.

The five-year survival in EOC is $\sim 45 \%$, owing to late diagnosis and lack of effective therapy (Lheureux et al., 2019). Yet in many cases, the initial response to therapy is good and accompanied by a drop of CA125, but relapse often occurs within 6-24 months, indicating the need for better indicators of therapeutic response. In our small study, $\mathrm{G} \mu \mathrm{F}$ revealed interesting differences among patients. Five patients (P1-P5) with serous cystadenocarcinoma (all metastatic) were analyzed. While for P1 a sample could be collected before chemotherapy, a time course study was conducted, with the treatment efficacy monitored by CA125. The number of scCTCs, cCTCs, and the size of cCTCs were significantly lower for patients having followed several cycles of chemotherapy, and were also reduced significantly for P1 after the third chemotherapy cycle. Interestingly, the number of scCTCs remained stable and only dropped after the $6^{\text {th }}$ chemotherapy cycle, whereas the concentration of CA125 had dropped rapidly. Thus, in line with previous observations supporting that CTC phenotype correlates with disease progression and recurrence in breast cancer (Yu et al., 2013), these results suggest that scCTCs and 
cCTCs could complement CA125 as an independent measure of response to therapy. The detection of CTCs in non-metastatic patients by $\mathrm{G} \mu \mathrm{F}$ suggests also possible application to early diagnosis, which is supported by a study by Guo et al., who found CTC counts to be more sensitive than CA125 to identify patients at high risk for ovarian cancer (Guo et al., 2018).

In conclusion, we established $\mathrm{G} \mu \mathrm{F}$ as a simple yet powerful method for isolating cCTCs and scCTCs following optimization, taking into consideration the break-up of cCTCs that we observed and quantified as function of flow rate. In particular, spiked and cCTCs were captured with $\sim 85 \%$ yield and released with $85 \%$ yield. cCTCs collected from patients and mouse models comprised between two to over one hundred cells. Our pilot study with 5 metastatic and 5 non-metastatic EOC patients (and 4 other metastatic cancer patients), found cCTCs in every patient and in some cases more cells were circulating as cCTCs than scCTCs. These results challenge commonly held notions that (i) cCTCs are only found rarely, (ii) only in low numbers, and (iii) constitute only a small fraction relative to scCTCs. To allow comparison between different studies and methods, the capture yield for scCTCs and cCTCs, cluster break-up, size distribution and composition of $\mathrm{cCTC}$ will need to be characterized. Effective isolation of cCTCs along with scCTCs will allow studying the role of CTCs in disease progression and metastasis, and their potential as surrogate biomarkers for diagnosis, prognosis and therapy monitoring.

\section{Acknowledgments}

We acknowledge funding from National Science and Engineering Research Council of Canada (NSERC) and CIHR (Canadian Institutes of Health Research) as part of the CHRP program (\#446671-13). D.J. acknowledges support from Canada Research Chair (CRC). J.A.H.-C. thanks the Lloyd Carr-Harris Foundation and CONACyT for fellowships. L.M., FRQS research scholar, thanks the CIHR for financial support. A-M.M-M. and D.P., researchers of the Centre de recherche du Centre Hospitalier de l'Université de Montréal, thank the FRQS and the Institut du Cancer de Montréal, and B.P. thanks the Institut TransMedTech for financial support. Tumor banking was supported by the Banque de tissus et données of the Réseau de recherche sur le cancer (RCC) of the Fond de recherche du Québec - Santé (FRQS), associated with the Canadian Tumor Repository Network (CTRNet). We gratefully acknowledge Dr. K. Rahimi from the department of pathology and cell biology at CRCHUM for advice 
and fruitful discussions. We thank Dr. Maïwenn Beaugrand and Adam Prager for their help in the data analysis of cluster growth in suspension.

\section{Author contributions}

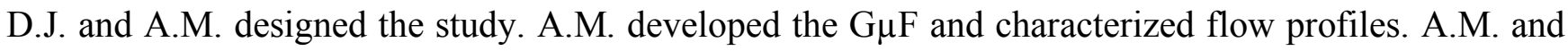
S.K. optimized the $\mathrm{G} \mu \mathrm{F}$ process using cell lines. With the expertise of T.V., J.A.H.-C. fabricated microfilters and conducted flow simulations. S.A.H., performed the orthotopic injection in mice. A.M. performed experiments with mouse and patient samples, including cell isolation, morphological and molecular characterization. A.M analyzed the data and wrote the manuscript with the assistance of D.J. L.M., D.P. and A-M.M-M. provided expertise in ovarian cancer and models. All authors discussed the results and commented on the manuscript.

\section{Declaration of Interests}

The authors declare no competing interests. 


\section{Figure legends}

Figure 1. G $\boldsymbol{\mu}$ F for CTC enrichment. (A) Schematic of the $\mathrm{G} \mu \mathrm{F}$ set-up. The total column height $\left(\mathrm{H}_{\text {tot }}\right)$ determines the pressure and flow rate when considering the $\mathrm{G} \mu \mathrm{F}$ flow resistance. (B) Exploded view of the filtration cartridge with filter, and close-ups of a polymer filter with $8 \mu \mathrm{m}$ diameter pores. (C) and (D) Examples of time course of flow rate for $10 \mathrm{~mL}$ of (C) PBS and (D) diluted blood filtered through an $8 \mu \mathrm{m}$ filter with $\mathrm{H}_{\text {tot }}=15 \mathrm{~cm}$, and closeup of domain II (pseudo-steady state) for diluted blood. See also Figures S1 and S2 and Tables S1 and S2.

Figure 2. G $\mu \mathrm{F}$ captured larger clusters than syringe-pump driven flow. (A) Fluorescence images of OV-90 single cells and clusters captured using $\mathrm{G} \mu \mathrm{F}$ and pump filtration using $8 \mu \mathrm{m}$ filters. Cells were stained for cytokeratin (green) and CD45 (red). Nuclei were stained with DAPI (blue). Single cells (white arrows), small clusters and WBCs (red arrow) were captured using both configurations. Larger clusters, with > 5-6 cells were only captured with $\mathrm{G} \mu \mathrm{F}$. (B) Capture efficiency of clusters and single cells for $\mathrm{G} \mu \mathrm{F}$ (green) and pump filtration (grey) at 0.1 and $0.5 \mathrm{~mL} \mathrm{~min}^{-1}$. (C) Size distribution of the clusters before filtration and captured using $\mathrm{G} \mu \mathrm{F}$ and pump filtration at $\sim 0.1 \mathrm{~mL} \mathrm{~min}^{-1}$. For each replicate, a known number of OV-90 cells ( $\sim 120$ clusters and $\sim 40$ single cells) was spiked in diluted blood. Error bars correspond to the standard deviation of three replicated experiments. (D) Finite-element analysis comparing flow velocity in $8 \mu \mathrm{m}$ pores for $\mathrm{G} \mu \mathrm{F}$ and pump filtration at $0.1 \mathrm{~mL} \mathrm{~min}^{-}$ ${ }^{1}$, for $0 \%$ and $22 \%$ clogging. See also Tables S1 and S3.

Figure 3. Characterization of clusters captured by $\mathbf{G} \boldsymbol{\mu F}$. (A) Fluorescence images of representative large and small clusters. Cells were stained for CK (green), CD45 (red) and with DAPI (blue). CTC-like cells (white arrow) are $\mathrm{CK}^{+} / \mathrm{CD} 45^{-} / \mathrm{DAPI}^{+}$and $\mathrm{WBCs}$ (red arrow) are $\mathrm{CK}^{-} / \mathrm{CD}^{2} 5^{+} / \mathrm{DAPI}^{+}$. (B) Capture efficiency depending on pore size, measured with OV-90 single cells (blue) and clusters (green) spiked in diluted blood. (C) Scatterplot and box plot of the size distribution of clusters captured by serial $\mathrm{G} \mu \mathrm{F}$ through filters with deceasing pore size $(28,20,15$, $12,10$ and $8 \mu \mathrm{m})$ for three replicated experiments $(\mathrm{n}=145,300$ and 550 spiked clusters). The boxes range from $25^{\text {th }}$ and $75^{\text {th }}$ percentiles, the whiskers correspond to $91^{\text {st }}$ and $9^{\text {th }}$ percentiles, and the horizontal lines represent the medians. (D) Cluster size distribution before filtration (dark grey, $\mathrm{n}=80,39$ and 37) and after filtration at $0.1 \mathrm{ml}$ $\min ^{-1}$ and release by flowing PBS at either at $0.1 \mathrm{~mL} \mathrm{~min}^{-1}$ (light grey, $\mathrm{n}=61,30$ and 35) or $1 \mathrm{~mL} \mathrm{~min}^{-1}$ (red, $\mathrm{n}=$ 37, 31 and 19). (E) Viablility of single cells (blue) and clusters (green) after filtration, rinsing, and release using complete OSE culture medium, PBS, or FBS at $4^{\circ} \mathrm{C}$, at RT $\left(22-23^{\circ} \mathrm{C}\right)$, and after $5 \mathrm{~h}$ incubation in low adhesion plates (no processsing) at $37^{\circ} \mathrm{C}$. Error bars correspond to the standard deviation of three replicated experiments. $p$ $<0.01: * * ; p<0.05: *$. See also Figure S3.

Figure 4. cCTCs captured from mouse. A. Examples of fluorescence images of OVCAR-3-GFP cCTCs captured from mouse blood. Nuclei were stained with DAPI. B. Size distribution of the OVCAR-3-GFP cCTCs captured in three replicates from a single mouse. cCTC area is averaged over three replicates. C. Evolution of the average area of OV-90 and OVCAR-3-GFP cCTCs overtime during incubation in low adhesion culture flasks. Error bars 
correspond to the standard deviation of three replicated experiments. D. Fluorescence images of OV-90 cCTCs captured from mice blood, and OVCAR-3 cCTCs from the blood and ascites of the same mouse. cCTCs were stained for aggressiveness markers E-Cad, ZO-1, ZEB1 and Snail. Nuclei were stained with DAPI. E. Expression levels of E-cad, ZO-1, ZEB-1 and Snail in OV-90 and OVCAR-3 cCTCs from blood or ascites. F. Additional staining for mesenchymal Vim. OVCAR-3 cCTCs from blood and ascites exhibit a hybrid E/M phenotype. Error bars correspond to the standard deviation between cCTCs. See also Figure S4.

Figure 5. Count and morphological characterization of CTCs captured from the blood of EOC patients with advanced high-grade serous carcinoma (left) and other types EOC at lower stage (right). (A-B) Concentration of scCTCs and cCTCs in the blood of patients P1 to 10. The diamonds represent the frequency of cCTC events. (C-D) Number of scCTCs and of cells in cCTCs per milliliter of blood, in each patient sample. The triangles correspond to the percentage of cells in cCTCs $v$. all CTCs. (E-F) Size distribution of cCTCs captured from the blood of P1 to P10. The area covered by cCTC clusters is provided (dots) on an indicative basis. Error bars correspond to the standard deviation between cCTC of same number of cells in each patient.

Figure 6. Time-course study for one metastatic HGSC patient. A. Time course study for P1 who responded to therapy, showing the CA125 concentration as well as the number of scCTC and cCTC events per mL of blood at different time points during chemotherapy. B. cCTCs size distribution and C. representative bright field images of cCTCs captured from the blood of P1 at four time points during follow-up.

\section{Tables}

Table 1. Patient information. The time at which blood was drawn and analyzed as well as the CA125 concentration in blood at the time of CTC analysis is provided. 


\section{Methods}

Materials and reagents. All solutions were prepared using water from a Milli-Q system (resistivity: 18 $\mathrm{M} \Omega \mathrm{cm}$; Millipore). Phosphate buffered saline (PBS, 1X, pH=7.4, Fisher Scientific), contains $11.910^{-3}$, $137.010^{-3}$ and $2.710^{-3}$ mol L-1 of phosphates $\left(\mathrm{Na}_{2} \mathrm{HPO}_{4}\right.$ and $\left.\mathrm{KH}_{2} \mathrm{PO}_{4}\right), \mathrm{NaCl}$ and $\mathrm{KCl}$, respectively. Trypsin-EDTA, bovine serum albumin (BSA), bovine insulin and Tween 20 were obtained from SigmaAldrich. Triton X-100 and paraformaldehyde (PFA) were purchased from Fisher Scientific. OSE (Ovarian surface epithelial) medium, L-glutamine and HEPES were obtained from Wisent. RPMI (Roswell Park Memorial Institute) 1640 medium, fetal bovine serum (FBS) and 4',6-diamidino-2phenylindole (DAPI) were purchased from Life Technologies. Antibiotics (penicillin/streptomycin) were obtained from Invitrogen. Anti-human CD45-PE (cluster of differentiation 45, Cat. \#FAB1430P), antiCK 18-AF 488 (cytokeratin 18, labeled with Alexa Fluor 488, Cat. \#IC7619G) and anti-E-Cad (Ecadherin, Cat. \#MAB18382, from mouse) were obtained from R\&D systems. Anti-Vim (Vimentin, Cat. \#SAB4503081, from rabbit), anti-ZO-1 (Zonula occludens-1, Cat. \#AB2272, from rabbit), anti-ZEB-1 (Zinc finger E-box-binding homeobox 1, Cat. \#SAB3500514, from rabbit), and anti-Snail (Drosophila embryonic protein, Cat. \#SAB2108482, from rabbit) were purchased from Sigma-Aldrich. AntiEpCAM-PE (anti-epithelial cell adhesion molecule labeled with phycoerythrin, Cat. \#12-9326-42), antic-MET-FITC (hepatocyte growth factor receptor, labeled with fluorescein isothiocyanate, Cat. \#11-885842), and detection antibodies goat anti-mouse-Alexa Fluor 647 (Cat. \#A21240) and goat anti-rabbit-Cy3 (Cyanine 3, Cat. \#A10520) were obtained from Fisher Scientific.

Filter fabrication. The filter fabrication process, extensively described elsewhere (Hernandez-Castro et al., 2017), allows for the fabrication of porous membrane with pore diameters of $8,10,12,15,20$ and 28 $\mu \mathrm{m}$, referred to as $\mathrm{X} \mu \mathrm{m}$ filters throughout the text. Briefly, pillar structures with a diameter corresponding to that of the pore to be created were prepared by standard photolithography and deep reactive-ion etching (DRIE). A UV-curable polymer cover coated on polyethylene terephthalate (PET) carrier was placed onto the pillars to close the structure. The formed cavity was then filled with Fluorolink ${ }^{\circledR}$ MD 700, which was then cured through UV exposure (2000-EC Series UV curing flood lamp, DYMAX). Finally, the blank cover was peeled off, and the molds were bathed in acetone for 15$20 \mathrm{~min}$, for the membranes to self-de-mold from the pillars. Filters consist of a 20-40 $\mu$ m-thick porous membrane heat bonded to a PMMA ring, which defines an $8 \mathrm{~mm}$-diameter filter. Before filtration, filter surface was passivated by incubation in BSA (2\% in PBS) to reduce non-specific adsorption on the filter. 
Filtration cartridge. The filtration cartridge $(70 \times 40 \mathrm{~mm})$ was designed with AutoCAD software (Autodesk Inc.) and 3D printed (Perfactory Micro EDU, Envision Tech) (Meunier et al., 2016). It consists of a top (10 mm high) and bottom (15 mm high) parts with notches to place the filter between toric joints and allow for its alignment with the inlet and outlet. A silicone gasket and a pair of screws and bolts are used for sealing.

Gravity-based microfiltration $(\mathbf{G} \boldsymbol{\mu F})$. The $\mathrm{G} \mu \mathrm{F}$ set-up consists of a $60 \mathrm{~mL}$ syringe (top reservoir) with its plunger removed, connected to the cartridge using a PEEK (Polyether ether ketone) tube (i.d. 0.75 $\mathrm{mm}$, Sigma Aldrich). The overall setup is immobilized using a retort stand and the inlet tube is clamped before pouring the sample into the top reservoir. In this $\mathrm{G} \mu \mathrm{F}$, the height of the fluid column determines the pressure, and consequently the flow rate. Flow rate calibration was performed for whole and diluted blood (1:6 (v/v) in PBS), as well as for PBS for various filter porosities and tube lengths. After the first seconds to minutes of filtration, flow rate stabilized and fluctuate around a unique value for one to two hours. Average flow rates were measured by collecting sample droplets at known time intervals (from few seconds to minutes) during filtration. The average flow rate, measured in this stable regime was found to increase linearly with the initial sample column height. For each fluid, calibration curves of the average flow rate as a function of the sample height were established and used to adjust the tube length to achieve a specific flow rate (Figure $\mathrm{S} 1$ and Table $\mathrm{S} 1$ ).

Cell culture. All culture media and solutions were sterile and filtered through a $0.2 \mu \mathrm{m}$ filter. The ovarian cancer OV90 cell line was developed in the laboratory of Drs. Provencher and Mes-Masson and has been well characterized (Provencher et al., 2000). It was established from the cellular fraction of a patient's ascites. OV90 cells were maintained in OSE medium, supplemented with 10\% FBS, 2\% L-glutamine, $1 \%$ HEPES, and 1\% (v/v) antibiotics (final concentrations of $100 \mathrm{I} . \mathrm{U} . \mathrm{mL}^{-1}$ penicillin and $100 \mu \mathrm{gL}^{-1}$ streptomycin). Ovarian cancer OVCAR-3 cells were cultured in RPMI 1640 medium supplemented with $20 \% \mathrm{FBS}, 1 \%(\mathrm{v} / \mathrm{v})$ penicillin/streptomycin, and $0.01 \mathrm{mg} \mathrm{mL}^{-1}$ bovine insulin. Cell lines were validated by short tandem repeat (STR) profiling. For both cell lines, adherent cells were observed releasing single cells and clusters in their surrounding medium. The culture medium, containing released clusters, was changed every 1-2 days, and when adherent cells formed almost confluent layers (80-90\%) in flasks, adherent cells were harvested using diluted trypsin. $200 \mu \mathrm{L}$ of the cell suspension was re-suspended in 5 $\mathrm{mL}$ of culture medium in a new flask. All cell cultures were maintained in $5 \% \mathrm{CO}_{2}$ at $37^{\circ} \mathrm{C}$ in $25 \mathrm{~cm}^{2}$ flasks (Corning). 
Spiking experiments with ovarian cell lines. The culture medium containing OV-90 clusters and very few single cells was harvested, centrifuged at $1300 \mathrm{rpm}$ for 5 minutes, and cells and clusters resuspended in PBS. Single cells were obtained by trypsinization of the cell monolayers. Then, OV-90 single cells were diluted in PBS to obtain approximately 20-50 cells per microliter. In order to precisely determine the number of single cells and clusters, $10 \mu \mathrm{L}$ droplets were placed between a microscope glass slide and a coverslip. The actual number of cells was manually counted twice on each slide and averaged on 10 droplets. Then, a known number of single cells and clusters was spiked in $1 \mathrm{~mL}$ blood samples. Blood was drawn from healthy volunteers (IRB study \#BMB-08-012) into $10 \mathrm{~mL}$ CTAD tubes (citrate-based anticoagulant containing the platelet inhibitors theophylline, adenosine, and bipyridamole, BD Vacutainer). Samples were maintained at $4{ }^{\circ} \mathrm{C}$ and processed within $72 \mathrm{~h}$ of collection.

Flow velocity simulations. Modeling studies were performed using the COMSOL Multiphysics software. Flow velocity profiles were obtained by 3D simulations through a cell of 9 pores. Filter clogging was simulated through the same cells with 2/9 closed pores. For G $\mu \mathrm{F}$ (constant pressure), inlet pressure was fixed and for pump filtration a constant flow rate was applied. Additional details are provided in Table S3.

Orthotopic mouse model of ovarian cancer. Female (8-12 weeks old) athymic nude mice (Crl:NU (NCr)-Foxn1nu; Charles River) were housed at the GCRC (Goodman Cancer Research Centre) animal facility and all procedures were conducted following ethics approval in accordance with the animal care guidelines at the Animal Resource Centre of McGill University. For orthotopic ovarian injections, $10 \mu \mathrm{L}$ of Geltrex (Invitrogen) containing $7.5 \times 10^{5}$ OV90 or OVCAR-3 cells as a single-cell suspension were injected into the ovary. No leakage from the injection site was observed. When specified, and prior to the injection, OVCAR-3 cells were fluorescently labeled by lentiviral transduction (OVCAR-3-GFP). Lentivirus was produced in HEK293LT cells, by transfection with the lentiviral transfer vector plasmid (pWPI) that contains enhanced green fluorescent protein (eGFP) (Addgene plasmid \# 12254), and provided by Dr. Didier Trono (Lausanne, Switzerland) (McCaffrey et al., 2012). Ovarian tumors formed in $\sim 6$ weeks and ascites were detected 8-10 weeks after injection. Animals that did not develop primary tumors, ascites, and metastases were excluded. Blood $(\sim 0.5 \mathrm{~mL})$ and ascites $(\sim 0.5-2 \mathrm{~mL})$ were collected under isoflurane anesthesia using a $23 \mathrm{G}$ needle and processed within 3-5 hours of collection.

Epithelial ovarian cancer patients. Blood and tumor samples from EOC patients were collected with informed consent from the Centre Hospitalier de l'Université de Montréal (CHUM), in the Division of Gynecologic Oncology (Table 1). This part of the study involving human samples was approved by both 
institutional ethics committees: the Comité d'éthique de la recherche du CHUM (CÉR-CHUM) and the McGill research ethics office (IRB study \#A05-M27-16B). Tumor stage was determined at time of surgery by a gynecologic oncologist. Histopathology and tumor grade were determined by a gynecological pathologist using criteria consistent with the International Federation of Gynecology and Obstetrics (FIGO) classification. Patient plasma CA125 levels is routinely measured during follow up. Blood samples used for CTC capture were kept at $4^{\circ} \mathrm{C}$ and processed within 6-14 hours of collection.

CTC capture. Before filtration, all samples were diluted 1:6 (v/v) in PBS. For filtration at constant flow rate using a syringe pump, samples were placed in a $10 \mathrm{~mL}$ syringe. For $\mathrm{G} \mu \mathrm{F}$, flow rate was adjusted by changing tube length based on calibration (Table S1). Initially, the inlet tube was clamped, and the sample was poured into the top reservoir, then filtration would start as the clamp was removed. Unless mentioned otherwise, filtration was performed at room temperature $\left(22-23^{\circ} \mathrm{C}\right)$ and after filtration samples were rinsed twice with $5 \mathrm{~mL}$ of PBS at the same flow rate as that of filtration.

Cell staining. Cell staining can be performed on the filter, directly in the cartridge after filtration as previously described (Meunier et al., 2016) or after release in a culture dish. Cells were fixed with 3.7\% paraformaldehyde (PFA), rinsed with PBS, permeabilized with $0.2 \%$ Triton $\mathrm{X}-100$, then rinsed again with PBS. Blocking was performed with 1.0\% BSA in PBS supplemented with $0.1 \%$ Tween 20. Then, for identification, cells were stained with anti-CK (cytokeratin) 18-Alexa Fluor $488\left(2.0 \mu \mathrm{g} \mathrm{mL}^{-1}\right)$ and anti-human CD45-PE (cluster of differentiation 45, labeled with phycoerythrin, $1.0 \mu \mathrm{g} \mathrm{mL}^{-1}$ ) to further sort cancer cells from blood cells. For characterization, CTCs from mouse blood and ascites were stained using anti-E-Cad, anti-Vim, anti-ZO-1, anti-ZEB-1, or anti-Snail (1 mg mL $\left.{ }^{-1}\right)$, and GAM-647 or GARCy3 $\left(4.0 \mu \mathrm{g} \mathrm{mL}^{-1}\right)$ for detection. Cells were rinsed with PBS, then their nucleus was counterstained with 4',6-diamidino-2-phenylindole (DAPI, $0.1 \mu \mathrm{g} \mathrm{mL} \mathrm{mL}^{-1}$ ).

Cell release. Once filtration was over, the cartridge was placed upside down and $5 \mathrm{~mL}$ of PBS (or another fluid, where mentioned) were flown by gravity. Flow rate was controlled by adjusting the tube length. The fluid passes through the filter from the outlet to the inlet, thus mechanically detaching cells from the filter. The cell suspension was then centrifuged (1300 rpm, $5 \mathrm{~min}$ ) and re-suspended in PBS before further staining or in culture medium for growth.

Cell viability. Single cell and cluster viability was characterized after processing (dilution, filtration and release) with ovarian surface epithelial (OSE) culture medium ( $\mathrm{n}=625 / 248,690 / 626$, and 508/186 single cells/clusters), phosphate buffer solution (PBS, $n=401 / 225,199 / 58$, and 474/238), or fetal bovine serum (FBS, $\mathrm{n}=542 / 415,384 / 184$, and 459/308) using a live/dead kit (Thermofisher, \#L3224). Cell 
suspensions were centrifuged, rinsed with PBS, centrifuged again and stained by incubation with 4.0 $\mu \mathrm{mol} \mathrm{L}{ }^{-1}$ of EthD-1 (red, dead cells) and $2.0 \mu \mathrm{mol} \mathrm{L}^{-1}$ of calcein AM (green, live cells) diluted in PBS for $45 \mathrm{~min}$ at room temperature. As a positive control, single cells and clusters, directly harvested from culture flasks, were seeded in ultra-low attachment well plates (Corning \#3473) to avoid cell adhesion and then incubated for 5 hours at $37^{\circ} \mathrm{C}$ in OSE, PBS, or FBS. For negative control, dead cells were prepared by incubation in $70 \%$ methanol for 45 minutes. Viability, was determined using fluorescence microscopy (excitation/emission wavelengths: 485/530 and 530/645 nm for calcein AM and EthD-1, respectively), corresponds to the ratio between the number of live single cells or clusters versus the total number of single cells or clusters per image, and was averaged over 5 images per condition and three replicated experiments.

Fluorescence microscopy. Filters were placed upside down on the platform of an inverted microscope (TE-2000-E, Nikon) connected to a CCD camera (QuantEM 512SC, Photometrics), and fluorescence images were recorded with NIS-Elements Advanced Research software (Nikon), and analyzed with ImageJ (W. Rasband). Images were collected with a mercury arc lamp and using 41001 (blue, for AF 488, GFP, FITC and Cy3), 41004 (green, for PE and AF647), and 31000v2 (UV for DAPI) filter cubes (Chroma Technology Corp.). Cells are defined as CTC(-like) when they have a nucleus (DAPI) and express CK, a cytoplasmic protein of epithelial origin. White blood cells (WBCs) also possess a nucleus but express CD45. For comparison and relative quantification of the expression levels of E-Cad, Vim, ZEB-1, ZO-1, Snail, EpCAM, and c-MET within different cluster models, same exposure time (1 second) was used for all images.

Migration assay. Growth of OVCAR-3 and OV-90 clusters, isolated from mouse blood, was evaluated using migration assays. About $510^{5}$ cells $\mathrm{mL}^{-1}$ were seeded in each well of 2 -well silicone inserts (Ibidi, Germany) placed on the bottom of a Petri dish. Cells were incubated overnight, then the silicon insert was removed. The free-cell area was imaged and averaged over 10 images at different time points. The closure of the free-cell area overtime (\% closure) was thus determined by comparison with the reference, measured right after removing the silicone inserts, and averaged over three replicated experiments (Figure S4A-D).

Sphere forming assay. After capture from mouse blood, OV-90 and OVCAR-3 clusters were resuspended in culture medium and incubated in ultra-low attachment wells. Spheres were grown for seven days, with gentle mixing once a day by pipetting. Samples were imaged right after seeding and over a few days of incubation using bright field microscopy. The area covered by clusters was measured using 
ImageJ software. Cluster proliferation in suspension was estimated by averaging the area of $\sim 400-500$ clusters over a few days and for three replicated experiments (Figure 4C). Evolution of the area distribution of OV-90 and OVCAR-3 clusters over time is presented in figure S4E-G.

Statistical analysis. The data are presented as mean \pm standard deviation measured over three replicates. Comparison of quantitative measures carried out on two independent groups was performed using unpaired two-tailed Student's t-tests. Statistical significance was set as $\mathrm{p}<0.05$.

\section{Supplemental Information}

All data generated or analyzed in this study are included in this published article and its supplementary information file.

\section{References}

Aceto, N., Bardia, A., Miyamoto, D. T., Donaldson, M. C., Wittner, B. S., Spencer, J. A., Yu, M., Pely, A., Engstrom, A., Zhu, H., et al. (2014). Circulating tumor cell clusters are oligoclonal precursors of breast cancer metastasis. Cell, 158, 1110-1122.

Adams, D. L., Zhu, P., Makarova, O. V., Martin, S. S., Charpentier, M., Chumsri, S., Li, S., Amstutz, P. \& Tang, C.-M. (2014). The systematic study of circulating tumor cell isolation using lithographic microfilters. RSC Adv., 4, 4334-4342.

Al Habyan, S., Kalos, C., Szymborski, J. \& McCaffrey, L. (2018). Multicellular detachment generates metastatic spheroids during intra-abdominal dissemination in epithelial ovarian cancer. Oncogene, 37, 5127-5135.

Allard, W. J., Matera, J., Miller, M. C., Repollet, M., Connelly, M. C., Rao, C., Tibbe, A. G., Uhr, J. W. \& Terstappen, L. W. (2004). Tumor cells circulate in the peripheral blood of all major carcinomas but not in healthy subjects or patients with nonmalignant diseases. Clin. Cancer Res., 10, 6897-6904.

Arfors, K.-E., Bergqvist, D., Intaglietta, M. \& Westergren, B. (1975). Measurements of blood flow velocity in the microcirculation. Ups. J. Med. Sci., 80, 27-33.

Ashworth, T. (1869). A case of cancer in which cells similar to those in the tumours were seen in the blood after death. Med. J. Aust., 14, 146-149.

Au, S. H., Edd, J., Stoddard, A. E., Wong, K. H. K., Fachin, F., Maheswaran, S., Haber, D. A., Stott, S. L., Kapur, R. \& Toner, M. (2017). Microfluidic isolation of circulating tumor cell clusters by size and asymmetry. Sci. Rep., 7, 2433.

Au, S. H., Storey, B. D., Moore, J. C., Tang, Q., Chen, Y.-L., Javaid, S., Sarioglu, A. F., Sullivan, R., Madden, M. W., O'Keefe, R., et al. (2016). Clusters of circulating tumor cells traverse capillary-sized vessels. Proc. Natl. Acad. Sci. U.S.A., 113, 4947-4952. 
Ballermann, B. J., Dardik, A., Eng, E. \& Liu, A. (1998). Shear stress and the endothelium. Kidney Int., 54, S100S108.

Brandt, B., Junker, R., Griwatz, C., Heidl, S., Brinkmann, O., Semjonow, A., Assmann, G. \& Zänker, K. S. (1996). Isolation of prostate-derived single cells and cell clusters from human peripheral blood. Cancer Res., 56, 45564561.

Cano, A., Perez-Moreno, M. A., Rodrigo, I., Locascio, A., Blanco, M. J., del Barrio, M. G., Portillo, F. \& Nieto, M. A. (2000). The transcription factor Snail controls epithelial-mesenchymal transitions by repressing Ecadherin expression. Nat. Cell Biol., 2, 76-83.

Cheng, S.-B., Xie, M., Chen, Y., Xiong, J., Liu, Y., Chen, Z., Guo, S., Shu, Y., Wang, M., Yuan, B., et al. (2017). Three-dimensional scaffold chip with thermosensitive coating for capture and reversible release of individual and cluster of circulating tumor cells. Anal. Chem., 89, 7924-7932.

Fan, T., Zhao, Q., Chen, J. J., Chen, W.-T. \& Pearl, M. L. (2009). Clinical significance of circulating tumor cells detected by an invasion assay in peripheral blood of patients with ovarian cancer. Gynecol. Oncol., 112, 185191.

Ferreira, M. M., Ramani, V. C. \& Jeffrey, S. S. (2016). Circulating tumor cell technologies. Mol. Oncol., 10, 374394.

Fleischer, R. L., Price, P. B. \& Symes, E. M. (1964). Novel filter for biological materials. Science, 143, 249-250.

Frisch, S. M. \& Francis, H. (1994). Disruption of epithelial cell-matrix interactions induces apoptosis. J. Cell Biol., 124, 619-626.

Guo, Y.-X., Neoh, K. H., Chang, X.-H., Sun, Y., Cheng, H.-Y., Ye, X., Ma, R.-Q., Han, R. P. \& Cui, H. (2018). Diagnostic value of HE4+ circulating tumor cells in patients with suspicious ovarian cancer. Oncotarget, 9, $7522-7533$.

Haslehurst, A. M., Koti, M., Dharsee, M., Nuin, P., Evans, K., Geraci, J., Childs, T., Chen, J., Li, J. \& Weberpals, J. (2012). EMT transcription factors snail and slug directly contribute to cisplatin resistance in ovarian cancer. BMC Cancer, 12, 91.

Hernandez-Castro, J. A., Li, K., Meunier, A., Juncker, D. \& Veres, T. (2017). Fabrication of large-area polymer microfilter membranes and their application for particle and cell enrichment. Lab Chip, 17, 1960-1969.

Holdich, R., Kosvintsev, S., Cumming, I. \& Zhdanov, S. (2006). Pore design and engineering for filters and membranes. Phil. Trans. R. Soc. A, 364, 161-174.

Hou, H. W., Warkiani, M. E., Khoo, B. L., Li, Z. R., Soo, R. A., Tan, D. S.-W., Lim, W.-T., Han, J., Bhagat, A. A. S. \& Lim, C. T. (2013). Isolation and retrieval of circulating tumor cells using centrifugal forces. Sci. Rep., 3,1259 .

Judson, P. L., Geller, M. A., Bliss, R. L., Boente, M. P., Downs, L. S. \& Argenta, P. A. (2003). Preoperative detection of peripherally circulating cancer cells and its prognostic significance in ovarian cancer. Gynecol. Oncol., 91, 389-394.

Kim, S., Kim, B. \& Song, Y. S. (2016). Ascites modulates cancer cell behavior, contributing to tumor heterogeneity in ovarian cancer. Cancer Sci., 107, 1173-1178.

Lecharpentier, A., Vielh, P., Perez-Moreno, P., Planchard, D., Soria, J. \& Farace, F. (2011). Detection of circulating tumour cells with a hybrid (epithelial/mesenchymal) phenotype in patients with metastatic nonsmall cell lung cancer. Br. J. Cancer, 105, 1338-1341.

Lengyel, E., Burdette, J., Kenny, H., Matei, D., Pilrose, J., Haluska, P., Nephew, K., Hales, D. \& Stack, M. (2014). Epithelial ovarian cancer experimental models. Oncogene, 33, 3619-3633. 
Lheureux, S., Gourley, C., Vergote, I. \& Oza, A. M. (2019). Epithelial ovarian cancer. The Lancet, 393, 12401253.

Lim, L. S., Hu, M., Huang, M. C., Cheong, W. C., Gan, A. T. L., Looi, X. L., Leong, S. M., Koay, E. S.-C. \& Li, M.-H. (2012). Microsieve lab-chip device for rapid enumeration and fluorescence in situ hybridization of circulating tumor cells. Lab Chip, 12, 4388-4396.

McCaffrey, L. M., Montalbano, J., Mihai, C. \& Macara, I. G. (2012). Loss of the Par3 polarity protein promotes breast tumorigenesis and metastasis. Cancer Cell, 22, 601-614.

Meunier, A., Hernández-Castro, J. A., Turner, K., Li, K., Veres, T. \& Juncker, D. (2016). Combination of mechanical and molecular filtration for enhanced enrichment of circulating tumor cells. Anal. Chem., 88, 85108517.

Ozkumur, E., Shah, A. M., Ciciliano, J. C., Emmink, B. L., Miyamoto, D. T., Brachtel, E., Yu, M., Chen, P.-i., Morgan, B., Trautwein, J., et al. (2013). Inertial focusing for tumor antigen-dependent and -independent sorting of rare circulating tumor cells. Sci. Transl. Med., 5, 179ra47-179ra47.

Pantel, K. \& Speicher, M. R. (2016). The biology of circulating tumor cells. Oncogene, 35, 1216-1224.

Polette, M., Mestdagt, M., Bindels, S., Nawrocki-Raby, B., Hunziker, W., Foidart, J. M., Birembaut, P. \& Gilles, C. (2007). $\beta$-Catenin and ZO-1: shuttle molecules involved in tumor invasion-associated epithelialmesenchymal transition processes. Cells Tissues Organs, 185, 61-65.

Poveda, A., Kaye, S. B., McCormack, R., Wang, S., Parekh, T., Ricci, D., Lebedinsky, C. A., Tercero, J. C., Zintl, P. \& Monk, B. J. (2011). Circulating tumor cells predict progression free survival and overall survival in patients with relapsed/recurrent advanced ovarian cancer. Gynecol. Oncol., 122, 567-572.

Provencher, D., Lounis, H., Champoux, L., Tetrault, M., Manderson, E., Wang, J., Eydoux, P., Savoie, R., Tonin, P. \& Mes-Masson, A.-M. (2000). Characterization of four novel epithelial ovarian cancer cell lines. In Vitro Cell. Dev. Biol. Animal, 36, 357-361.

Reddy, R. M., Murlidhar, V., Zhao, L., Grabauskiene, S., Zhang, Z., Ramnath, N., Lin, J., Chang, A. C., Carrott, P., Lynch, W., et al. (2016). Pulmonary venous blood sampling significantly increases the yield of circulating tumor cells in early-stage lung cancer. J. Thorac. Cardiovasc. Surg., 151, 852-858.

Ripperger, S., Gösele, W., Alt, C. \& Loewe, T. 2012. Filtration, 1. Fundamentals. Ullmann's Encyclopedia of Industrial Chemistry. Wiley-VCH Verlag GmbH \& Co. KGaA.

Sarioglu, A. F., Aceto, N., Kojic, N., Donaldson, M. C., Zeinali, M., Hamza, B., Engstrom, A., Zhu, H., Sundaresan, T. K., Miyamoto, D. T., et al. (2015). A microfluidic device for label-free, physical capture of circulating tumor cell clusters. Nat. Methods, 12, 685-691.

Seal, S. H. (1964). A sieve for the isolation of cancer cells and other large cells from the blood. Cancer, 17, 637642.

Stott, S. L., Hsu, C.-H., Tsukrov, D. I., Yu, M., Miyamoto, D. T., Waltman, B. A., Rothenberg, S. M., Shah, A. M., Smas, M. E. \& Korir, G. K. (2010). Isolation of circulating tumor cells using a microvortex-generating herringbone-chip. Proc. Natl. Acad. Sci. U.S.A., 107, 18392-18397.

Tan, S. J., Yobas, L., Lee, G. Y. H., Ong, C. N. \& Lim, C. T. (2009). Microdevice for the isolation and enumeration of cancer cells from blood. Biomed. Microdevices, 11, 883-892.

Tang, Y., Shi, J., Li, S., Wang, L., Cayre, Y. E. \& Chen, Y. (2014). Microfluidic device with integrated microfilter of conical-shaped holes for high efficiency and high purity capture of circulating tumor cells. Sci. Rep., 4, 6052. 
Wang, C., Mu, Z., Chervoneva, I., Austin, L., Ye, Z., Rossi, G., Palazzo, J. P., Sun, C., Abu-Khalaf, M. \& Myers, R. E. (2017). Longitudinally collected CTCs and CTC-clusters and clinical outcomes of metastatic breast cancer. Breast Cancer Res. Treat., 161, 83-94.

Wang, Y.-L., Zhao, X.-M., Shuai, Z.-F., Li, C.-Y., Bai, Q.-Y., Yu, X.-W. \& Wen, Q.-T. (2015). Snail promotes epithelial-mesenchymal transition and invasiveness in human ovarian cancer cells. Int. J. Clin. Exp. Med., 8, 7388-7393.

Yu, M., Bardia, A., Wittner, B. S., Stott, S. L., Smas, M. E., Ting, D. T., Isakoff, S. J., Ciciliano, J. C., Wells, M. N., Shah, A. M., et al. (2013). Circulating breast tumor cells exhibit dynamic changes in epithelial and mesenchymal composition. Science, 339, 580-584. 